\title{
Modeling and Optimization of Adsorption of Heavy Metal Ions onto Local Activated Carbon
}

\author{
Abbas Sabah Thajeel \\ Chemical Engineering Department, College of Engineering, University of Basrah \\ Basrah, Iraq \\ E-mail: abbas_amery2002@yahoo.com
}

Received: April 22, 2013 Accepted: June 1, 2013 Published: July1, 2013

doi:10.5296/ast.v1i2.3890 URL: http://dx.doi.org/10.5296/ast.v1i2.3890

\begin{abstract}
In this study, a mathematical model was constructed according toCentral Composite Design method (CCD), which simulated the experimental work for adsorption of $\left(\mathrm{Cu}^{2+}, \mathrm{Fe}^{3+}, \mathrm{Pb}^{2+}\right.$ and $\mathrm{Zn}^{2+}$ ) in batch adsorption processes, where this model was studied the different effects of operational conditions and their impact on the efficiency of adsorption by using activated carbon produced from rice husk as local raw material which is low cost and available in huge quantities, and find a final form simulates practical experiences. Finally a mathematical model has been used as a software program (minitab16).
\end{abstract}

Keyword: Activated carbon, Adsorption, Modeling, Design of experiments, Heavy metal ions, Rice husk, Central composite design 


\section{Introduction}

Heavy metal ions such as copper, iron, nickel, lead, etc. in the environment are of major concern due to their toxicity to many life forms. Unlike organic pollutants, which are susceptible to biological degradation, metal ions do not degrade into any harmless end products (Mohammadiet al., 2010) and tend to accumulate causing several diseases and health disorders in humans, and other living organisms (Rosa et al., 2008). Several industrial activities are important sources ofenvironmental pollution due to their high content of several heavy metal ions(Dada et al., 2012). Wide range of various treatment techniques available for the removal of heavy metal ions from aqueous solutions such as ion exchange, biodegradation, oxidation, solvent extraction, chemical precipitation, flotation, biosorption, electrolytic recovery, membrane separation and adsorption have been reported to be used for removal of heavy metal ions from industrialeffluents (Al-tameemi et al., 2012; Deosarkar,2012). However, adsorption has been universally accepted as one of the most effective pollutant removal process, with low cost, ease in handling, low consumption of reagents, as well as scope for recovery of value added components through desorption and regeneration of adsorbent(Dada et al., 2012). Adsorption is collection of adsorbate on the surface of adsorbent due to force of attraction(Deosarkar,2012). The practical applications of adsorption can be at separation and purification of liquid and gas mixtures, bulk chemicals, drying gases and liquids before loading them into industrial systems, removal of impurities from liquid and gas media, recovery of chemicals from industrial and vent gases and water purification(Prabakaran\&Arivoli,2012). Activated carbon is the most widely used adsorbent due to its excellent adsorption capability for heavy metals. However, the use of these methods is often limited due to the high cost, which makes them unfavorable for the needs of developing countries. Many reports have been investigatedthe low-cost adsorbents for Adsorption of heavy metals from aqueous solutions(Souag et al., 2009)such as date pits(Belhachemi et al., 2009) bamboo(Kannan\&Veemaraj, 2009) oil palm fibre(Hameedet al., 2011; Nwabanne\&Igbokwe, 2012), coconut shell(Satya et al., 1997), apricot stones (Philip\&Girgis, 1996), sugar beet bagasse (Jaguaribe et al., 2000), waste tires(Teng et al., 2000;Juan et al., 2005; Mui et al., 2010), coconut husk, seed shell (Gueu et al.,2006), dates stones (Alhamed\&Bamufleh, 2008), sun flower (Surchi, 2011), asphaltic carbon(Ambursa et al., 2011), Henna Leaves (Shanthi\&Selvarajan, 2012). The intrinsic properties of activated carbon are dependent on the raw material source. The source of raw material was based on the need for developing low cost absorbent for pollution control as well as reducing the effect of environmental degradation poised by agricultural waste(Itodo H. \&Itodo A., 2010).

In this study, a simulation of batch adsorption processes was investigated by mathematical model for adsorption of heavy metal ions such as $\left(\mathrm{Fe}^{3+}, \mathrm{Zn}^{2+}, \mathrm{Cu}^{2+}\right.$ and $\left.\mathrm{Pb}^{2+}\right)$ from the (oil-water)polluted which comes out from the oil industry in Basrah cityonto activated carbon produced from rice husk (RHAC) as local raw material which is low cost and available in huge quantities causing a pollutant problem. 


\section{Experimental Section}

\subsection{Materials}

Zinc chloride with purity (97\%) and sodium hydroxide with purity $(97.5 \%)$ were supplied from THOMAS BAKER (Chemicals) Company. Copper chloride anhydrous with purity (99\%) was supplied from BDH(Chemicals) Company. Iron nitrate (ferric nitrate) with purity (99\%) was supplied from MERCK Company. Hydrogen chloride with purity $(37 \%)$ was supplied from Scharlab.S.L Company. Nitrogen gas with purity (90\%)and carbon dioxide gases with purity (95 - $99 \%$ )were supplied from Basrah Factory. Rice husks were collected from Almshgab City Al-najafALashraf, Iraq, which had been discarded as waste from rice cultivation.

\subsection{Adsorbent}

Activated carbon produced from rice husk (RHAC) by physical method was used as an adsorbent material in this study, the preparation method was described following:

Initially, the (RH) were well washed with distilled water and dried in electrical oven for 24 hours. The carbonization step was carried out in electrical furnace for $2 \mathrm{hr}$ at $500 \dot{\mathrm{C}}$ and heating rate of $30^{\circ} \mathrm{C} / \mathrm{min}$ in absence air using nitrogen $\left(\mathrm{N}_{2}\right)$ at flow rate is $200 \mathrm{~L} / \mathrm{min}$.

In the activation step, the product from carbonization step was activated by passing carbon dioxide $\left(\mathrm{CO}_{2}\right)$ instead of nitrogen for $2 \mathrm{hr}$ at $700 \dot{\mathrm{C}}$.

\subsection{Preparation of Standard Solutions}

The stock solutions of $1000 \mathrm{mg} / \mathrm{L}(\mathrm{ppm})$ of $\mathrm{Cu}^{2+}, \mathrm{Fe}^{3+}, \mathrm{Pb}^{2+}$ and $\mathrm{Zn}^{2+}$ were prepared by dissolving $2.1368 \mathrm{~g}$ of $\mathrm{CuCl}_{2}, 7.3073 \mathrm{~g}$ ofFe $\left(\mathrm{NO}_{3}\right)_{3}, 1.3557 \mathrm{~g}$ of $\mathrm{PbCl}_{2}$ and $2.1273 \mathrm{~g}$ of $\mathrm{ZnCl}_{2}$ in $1000 \mathrm{ml}$ volumetric flasks and fill up to the mark with distilled water.

The diluted concentrations were prepared from stock solutions for carrying out experiments.

A certain volume $(10 \mathrm{ml})$ of oil has been added to all above solutions with efficient agitation for simulated waste oil water.

\subsection{Analyze a Sample of Heavy Metal Ions by Using Atomic Absorption Spectrophotometer $(A A S)$}

The concentration of metal ions was measured by using atomic absorption spectrophotometer (BUCK Scientific, Model 210 VGP). In atomic absorption spectroscopy, metal atoms were vaporized into a flame, and the metal vapor absorbed radiation from the specific hollow cathode lamp in proportion to the number of atoms present. Beer's Law was followed in the part-per-million range (remember that ppm means $\mathrm{mg}$ of metal/liter of solution).

\subsection{Adsorption Studies}

Batch experiments were carried out by a $(125 \mathrm{ml})$ flask. A certain weight of adsorbent material and $(100 \mathrm{ml})$ of the solution prepared previously were added to the flask, and installed in the water bath (MemmertGmbh Type WMB 22) at different temperature, see 


\section{Macrothink}

Figure1. The $\mathrm{pH}$ values were controlling by adding $0.1 \mathrm{~N} \mathrm{NaOH}$ or $0.1 \mathrm{~N} \mathrm{HCl}$.A mixture with a different speed was mixed for 15 minutes using Variable-Speed Benchtop, model 5850, Eberbach. Finally, the mixtures were filtered through filter paper, and measurement of concentrations by Atomic Absorption.

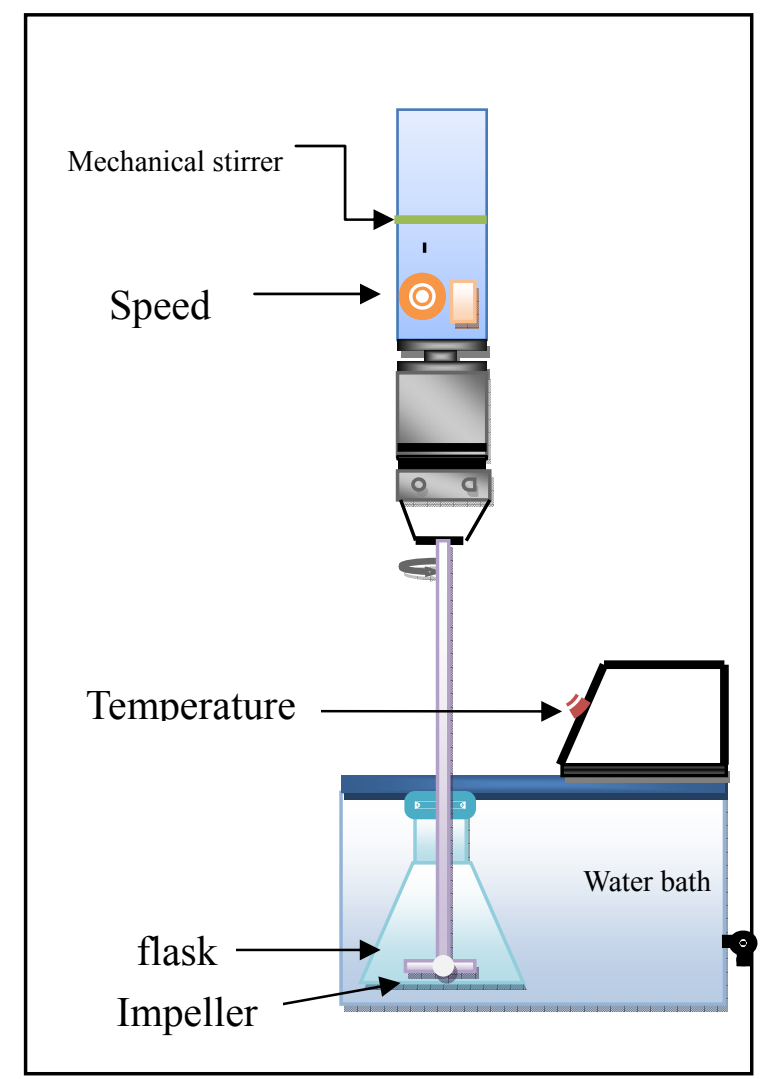

Figure 1. Schematic diagram of the batch adsorber (Al-Jomaa, 2011)

The removal percentage (R.P.\%) which described the efficiency of adsorbent to adsorbed a heavy metal ions is calculated by following equation (BADMUS et al., 2007; Itodo et al., 2010):

$$
\text { R.P. \% }=\frac{C_{i}-C_{f}}{C_{i}} * 100
$$

Where: $C_{i}$ and $C_{f}$ are the initial and final concentration in (ppm), respectively.

\section{Results and Discussion}

\subsection{Modeling of Heavy Metal Ions Adsorption}

Design of experiments (DOE) methods all involve: (1) carefully planning sets of input combinations to test using a random run order; then, (2) tests are performed and output values are recorded; (3) an interpolation method such as "regression" is then used to interpolate the outputs; and (4) the resulting prediction model is then used to predict new outputs for new 
possible input combinations, DOE methods can be an important part of systemoptimization.These methods all involve the activities of experimental planning, conducting experiments, and fitting models to the outputs(Allen, 2006).

DOE methods are classified into several types, which included screening using fractional factorials, response surface methods (RSM), and robust design procedures.All of these DOE methods involve changing key input variable settings which are directly controllable (called factors) using carefully planned patterns, and then observing outputs (called responses) (Allen, 2006).

Response surface methodology is a collection of statistical and mathematical methods that are useful for the modeling and analyzing engineering problems. In this technique, the main objective is to optimize the response surface that is influenced by various process parameters. Response surface methodology also quantifies the relationship between the controllable input parameters and the obtained response surfaces (Tanet al., 2007; Aslan \& Cebeci, 2007).

The particular value of the variable is called the level of the factor. The combination of factors used in a particular experiment is called a treatment (Al-Badran, 2003; Ghadeer, 2009).

RSM methods are based on three types of design of experiments (DOE) matrices. First, central composite designs (CCD) are matrices corresponding to (at most) five level experimental plans from Box and Wilson (1951). Second, Box Behnken designs (BBD) are matrices corresponding to three level experimental plans from Box, Behnken (1960). Third, Allen et al. (2003) proposed methods based on so-called expected integrated mean squared error optimal (EIMSE-optimal) designs (Allen, 2006).

In this study, the central composite design was used to determine a models which described therelationship between the variables and the response.

The response which is the product (Y), is assumed to be a random variable (Lazic, 2004).

$$
\mathrm{Y}=\mathrm{f}\left(\mathrm{X}_{1}, \mathrm{X}_{2}, \mathrm{X}_{3}\right)+\text { Error }
$$

Asecond degree polynomial equation was used if there is a curvature in the system, which given by Eq.(3) (Chen et al., 2011; Song et al., 2012; Daffalla et al., 2012):

$$
\mathrm{Y}_{\mathrm{b}}=\beta_{\circ}+\sum_{\mathrm{i}=1}^{\mathrm{k}} \beta_{\mathrm{i}} \mathrm{X}_{\mathrm{i}}+\sum_{\mathrm{i}=1}^{\mathrm{k}} \beta_{\mathrm{ii}} \mathrm{X}_{\mathrm{i}}^{2}+\sum_{\mathrm{i}<j}^{\mathrm{k}} \sum_{\mathrm{j}}^{\mathrm{k}} \beta_{\mathrm{ij}} \mathrm{X}_{\mathrm{i}} \mathrm{X}_{\mathrm{j}}
$$

Where:

$\mathrm{Y}_{\mathrm{b}}, \mathrm{X}_{\mathrm{i}}, \beta_{\circ}, \beta_{\mathrm{i}}, \beta_{\mathrm{ii}}, \beta_{\mathrm{ij}}$ and $X_{i, j}$ arethe predicted response, independent variables, model constant,linear coefficients, the quadratic coefficients and cross product coefficients, the coded values of variables, respectively.

These second -order designs for $\mathrm{k}$ factors are composed of three sets of points (John, 1998):

(i) $A 2^{\mathrm{K}}$ factorial design with $\mathrm{X}_{\mathrm{i}}= \pm 1$, these are called the cube points. There are $\mathrm{n}_{\mathrm{j}}$ of term. 
(ii) A set of axial points. There are $\mathrm{n}_{\alpha}=2 \mathrm{k}$. There coordinates are $( \pm \propto, 0,0, \ldots),(\ldots, 0, \pm \propto$ $, 0, \ldots)$ and $(\ldots, 0,0, \pm \alpha)$, where $\alpha$ is the distance of the axial point from center and makes the design rotatable(Tanet al., 2007).

(iii) $\mathrm{n}_{0}$ center points, which used to determine the experimental error and the reproducibility of the data.

The number of experiments $(\mathrm{N})$ needed was calculated by thefollowing equation (Lazic, 2004; John, 1998):

$$
N=n_{j}+n_{\alpha}+n_{0}=2^{k}+2 * k+n_{0}
$$

For five factors a second order polynomial mathematical model is describe by the following equation:

$$
\begin{gathered}
Y_{b}=\beta_{\circ}+\beta_{1} X_{1}+\beta_{2} X_{2}+\beta_{3} X_{3}+\beta_{4} X_{4}+\beta_{5} X_{5}+\beta_{11} X_{1}{ }^{2}+\beta_{22} X_{2}{ }^{2}+\beta_{33} X_{3}{ }^{2}+\beta_{44} X_{4}{ }^{2}+ \\
\beta_{55} X_{5}{ }^{2}+\beta_{12} X_{1} X_{2}+\beta_{13} X_{1} X_{3}+\beta_{14} X_{1} X_{4}+\beta_{15} X_{1} X_{5}+\beta_{23} X_{2} X_{3}+\beta_{24} X_{2} X_{4}+\beta_{25} X_{2} X_{5}+ \\
\beta_{23} X_{3} X_{4}+\beta_{35} X_{3} X_{5}+\beta_{45} X_{4} X_{5}
\end{gathered}
$$

The relationship between the coded levels and the corresponding actual variables is represented by the equation:

$$
X_{\text {coded }}=\frac{\left(x_{\text {actual }}-x_{\text {center }}\right)}{\left(x_{\text {center }}-x_{\text {minimum }}\right)}
$$

The low and height values of each variable in batch systemwere listed in Table 1:

Table 1. The low and height values of variables in batch system

\begin{tabular}{lcll}
\hline Variable & Simple & Low & Height \\
\hline PH & $X_{1}$ & 5 & 9 \\
TemperatureC & $X_{2}$ & 22 & 50 \\
Doseg & $X_{3}$ & 2 & 4 \\
Concentration ppm & $X_{4}$ & 21 & 50 \\
rpm & $X_{5}$ & 350 & 750 \\
\hline
\end{tabular}

The relationship between the coded variable $(\mathrm{X})$ and the corresponding real variable $(\mathrm{x})$ as following :

$$
\begin{gathered}
X_{1}=\frac{\left(x_{1}-7\right)}{2}, X_{2}=\frac{\left(x_{2}-36\right)}{14}, X_{3}=\frac{\left(x_{3}-3\right)}{1} \\
X_{4}=\frac{\left(x_{4}-35.5\right)}{14.5}, X_{5}=\frac{\left(x_{5}-550\right)}{200}
\end{gathered}
$$

The central composite design can be made to be rotatable by choosing $\alpha=2^{K / 4}$ when a complete factorial is used.For five factors:

$$
\alpha=2^{5 / 4}=2.378
$$




\section{Macrothink}

Aquatic Science and Technology

ISSN 2168-9148

2013, Vol. 1, No. 2

Table 2 . The coded \& uncoded (actual)values of variables in batch system

\begin{tabular}{|c|c|c|c|c|c|c|c|c|c|c|}
\hline No. & $\mathrm{X} 1$ & $\mathrm{X} 2$ & $\mathrm{X3}$ & $\mathrm{X4}$ & $\mathrm{X5}$ & $\mathrm{X} 1 \mathrm{PH}$ & X2Temp. & X3dose & X5CONC. & $\mathrm{X} 5 \mathrm{rpm}$ \\
\hline 1 & 1 & 1 & 1 & 1 & 1 & 9 & 50 & 4 & 50 & 750 \\
\hline 2 & -1 & 1 & 1 & 1 & 1 & 5 & 50 & 4 & 50 & 750 \\
\hline 3 & 1 & -1 & 1 & 1 & 1 & 9 & 22 & 4 & 50 & 750 \\
\hline 4 & -1 & -1 & 1 & 1 & 1 & 5 & 22 & 4 & 50 & 750 \\
\hline 5 & 1 & 1 & -1 & 1 & 1 & 9 & 50 & 2 & 50 & 750 \\
\hline 6 & -1 & 1 & -1 & 1 & 1 & 5 & 50 & 2 & 50 & 750 \\
\hline 7 & 1 & -1 & -1 & 1 & 1 & 9 & 22 & 2 & 50 & 750 \\
\hline 8 & -1 & -1 & -1 & 1 & 1 & 5 & 22 & 2 & 50 & 750 \\
\hline 9 & 1 & 1 & 1 & -1 & 1 & 9 & 50 & 4 & 21 & 750 \\
\hline 10 & -1 & 1 & 1 & -1 & 1 & 5 & 50 & 4 & 21 & 750 \\
\hline 11 & 1 & -1 & 1 & -1 & 1 & 9 & 22 & 4 & 21 & 750 \\
\hline 12 & -1 & -1 & 1 & -1 & 1 & 5 & 22 & 4 & 21 & 750 \\
\hline 13 & 1 & 1 & -1 & -1 & 1 & 9 & 50 & 2 & 21 & 750 \\
\hline 14 & -1 & 1 & -1 & -1 & 1 & 5 & 50 & 2 & 21 & 750 \\
\hline 15 & 1 & -1 & -1 & -1 & 1 & 9 & 22 & 2 & 21 & 750 \\
\hline 16 & -1 & -1 & -1 & -1 & 1 & 5 & 22 & 2 & 21 & 750 \\
\hline 17 & 1 & 1 & 1 & 1 & -1 & 9 & 50 & 4 & 50 & 350 \\
\hline 18 & -1 & 1 & 1 & 1 & -1 & 5 & 50 & 4 & 50 & 350 \\
\hline 19 & 1 & -1 & 1 & 1 & -1 & 9 & 22 & 4 & 50 & 350 \\
\hline 20 & -1 & -1 & 1 & 1 & -1 & 5 & 22 & 4 & 50 & 350 \\
\hline 21 & 1 & 1 & -1 & 1 & -1 & 9 & 50 & 2 & 50 & 350 \\
\hline 22 & -1 & 1 & -1 & 1 & -1 & 5 & 50 & 2 & 50 & 350 \\
\hline 23 & 1 & -1 & -1 & 1 & -1 & 9 & 22 & 2 & 50 & 350 \\
\hline 24 & -1 & -1 & -1 & 1 & -1 & 5 & 22 & 2 & 50 & 350 \\
\hline 25 & 1 & 1 & 1 & -1 & -1 & 9 & 50 & 4 & 21 & 350 \\
\hline 26 & -1 & 1 & 1 & -1 & -1 & 5 & 50 & 4 & 21 & 350 \\
\hline 27 & 1 & -1 & 1 & -1 & -1 & 9 & 22 & 4 & 21 & 350 \\
\hline 28 & -1 & -1 & 1 & -1 & -1 & 5 & 22 & 4 & 21 & 350 \\
\hline 29 & 1 & 1 & -1 & -1 & -1 & 9 & 50 & 2 & 21 & 350 \\
\hline 30 & -1 & 1 & -1 & -1 & -1 & 5 & 50 & 2 & 21 & 350 \\
\hline 31 & 1 & -1 & -1 & -1 & -1 & 9 & 22 & 2 & 21 & 350 \\
\hline 32 & -1 & -1 & -1 & -1 & -1 & 5 & 22 & 2 & 21 & 350 \\
\hline 33 & $-\alpha$ & 0 & 0 & 0 & 0 & 2.243 & 36 & 3 & 35.5 & 550 \\
\hline 34 & $\alpha$ & 0 & 0 & 0 & 0 & 11.76 & 36 & 3 & 35.5 & 550 \\
\hline 35 & 0 & $-\alpha$ & 0 & 0 & 0 & 7 & 2.702 & 3 & 35.5 & 550 \\
\hline 36 & 0 & $\alpha$ & 0 & 0 & 0 & 7 & 69.3 & 3 & 35.5 & 550 \\
\hline 37 & 0 & 0 & $-\alpha$ & 0 & 0 & 7 & 36 & 0.6216 & 35.5 & 550 \\
\hline 38 & 0 & 0 & $\alpha$ & 0 & 0 & 7 & 36 & 5.3784 & 35.5 & 550 \\
\hline 39 & 0 & 0 & 0 & $-\alpha$ & 0 & 7 & 36 & 3 & 1.013 & 550 \\
\hline 40 & 0 & 0 & 0 & $\alpha$ & 0 & 7 & 36 & 3 & 69.99 & 550 \\
\hline 41 & 0 & 0 & 0 & 0 & $-\alpha$ & 7 & 36 & 3 & 35.5 & 74.32 \\
\hline 42 & 0 & 0 & 0 & 0 & $\alpha$ & 7 & 36 & 3 & 35.5 & 1026 \\
\hline 43 & 0 & 0 & 0 & 0 & 0 & 7 & 36 & 3 & 35.5 & 550 \\
\hline 44 & 0 & 0 & 0 & 0 & 0 & 7 & 36 & 3 & 35.5 & 550 \\
\hline 45 & 0 & 0 & 0 & 0 & 0 & 7 & 36 & 3 & 35.5 & 550 \\
\hline 46 & 0 & 0 & 0 & 0 & 0 & 7 & 36 & 3 & 35.5 & 550 \\
\hline 47 & 0 & 0 & 0 & 0 & 0 & 7 & 36 & 3 & 35.5 & 550 \\
\hline 48 & 0 & 0 & 0 & 0 & 0 & 7 & 36 & 3 & 35.5 & 550 \\
\hline 49 & 0 & 0 & 0 & 0 & 0 & 7 & 36 & 3 & 35.5 & 550 \\
\hline 50 & 0 & 0 & 0 & 0 & 0 & 7 & 36 & 3 & 35.5 & 550 \\
\hline 51 & 0 & 0 & 0 & 0 & 0 & 7 & 36 & 3 & 35.5 & 550 \\
\hline 52 & 0 & 0 & 0 & 0 & 0 & 7 & 36 & 3 & 35.5 & 550 \\
\hline
\end{tabular}




\section{Macrothink}

The regression coefficients are determined by equations (Lazic, 2004):

$$
\begin{gathered}
\beta_{\circ=a_{1}} \sum_{1}^{N} Y_{b}-a_{2} \sum_{1}^{K} \sum_{1}^{N} X_{i}^{2} Y_{b} \\
\beta_{i=a_{3}} \sum_{1}^{N} X_{i} Y_{b} \\
\beta_{i j=a_{4}} \sum_{1}^{N} X_{i} X_{j} Y_{b} \\
\beta_{i i=a_{5}} \sum_{1}^{N} X_{i}^{2} Y_{b}+a_{6} \sum_{1}^{K} \sum_{1}^{N} X_{i}^{2} Y_{b}-a_{7} \sum_{1}^{N} Y_{b}
\end{gathered}
$$

Where: $a_{1} \ldots \ldots a_{7}$ are coefficients as determined from Table 3.

Table 3.Coefficients values $a_{1} \ldots \ldots . a_{7}$

\begin{tabular}{lllllll}
0.0988 & 0.0191 & 0.0231 & 0.0312 & 0.0156 & 0.0015 & 0.0191 \\
\hline
\end{tabular}

The results of experimental work of adsorption $\mathrm{Cu}^{2+}, \mathrm{Fe}^{3+}, \mathrm{Pb}^{2+}$ and $\mathrm{Zn}^{2+}$ ions onto RHACin batch processes are listed in Table A.1 Appendix A.

For $\mathrm{Cu}^{2+}$ ions were adsorbed on the RHAC in batch process:

Table 4. The calculated regression coefficients for $\mathrm{Cu}^{2+}$ ions

\begin{tabular}{lllllllll}
\hline No. & \multicolumn{1}{c}{$\boldsymbol{Y}_{\boldsymbol{b}}$} & \multicolumn{1}{c}{$\boldsymbol{X}_{\mathbf{1}} \boldsymbol{Y}_{\boldsymbol{b}}$} & \multicolumn{1}{c}{$\boldsymbol{X}_{\mathbf{2}} \boldsymbol{Y}_{\boldsymbol{b}}$} & $\boldsymbol{X}_{\mathbf{3}} \boldsymbol{Y}_{\boldsymbol{b}}$ & $\boldsymbol{X}_{\mathbf{4}} \boldsymbol{Y}_{\boldsymbol{b}}$ & $\boldsymbol{X}_{\mathbf{5}} \boldsymbol{Y}_{\boldsymbol{b}}$ & $\boldsymbol{X}_{\mathbf{1}} \boldsymbol{X}_{\mathbf{2}} \boldsymbol{Y}_{\boldsymbol{b}}$ & $\boldsymbol{X}_{\mathbf{1}} \boldsymbol{X}_{\mathbf{3}} \boldsymbol{Y}_{\boldsymbol{b}}$ \\
\hline 1 & 81.23938 & 81.23938 & 81.23938 & 81.23938 & 81.23938 & 81.23938 & 81.23938 & 81.23938 \\
2 & 84.54624 & -84.5462 & 84.54624 & 84.54624 & 84.54624 & 84.54624 & -84.5462 & -84.5462 \\
3 & 74.00122 & 74.00122 & -74.0012 & 74.00122 & 74.00122 & 74.00122 & -74.0012 & 74.00122 \\
4 & 76.60166 & -76.6017 & -76.6017 & 76.60166 & 76.60166 & 76.60166 & 76.60166 & -76.6017 \\
5 & 69.63233 & 69.63233 & 69.63233 & -69.6323 & 69.63233 & 69.63233 & 69.63233 & -69.6323 \\
6 & 73.71796 & -73.718 & 73.71796 & -73.718 & 73.71796 & 73.71796 & -73.718 & 73.71796 \\
7 & 64.84563 & 64.84563 & -64.8456 & -64.8456 & 64.84563 & 64.84563 & -64.8456 & -64.8456 \\
8 & 67.21829 & -67.2183 & -67.2183 & -67.2183 & 67.21829 & 67.21829 & 67.21829 & 67.21829 \\
9 & 90.37154 & 90.37154 & 90.37154 & 90.37154 & -90.3715 & 90.37154 & 90.37154 & 90.37154 \\
10 & 92.9487 & -92.9487 & 92.9487 & 92.9487 & -92.9487 & 92.9487 & -92.9487 & -92.9487 \\
11 & 85.91279 & 85.91279 & -85.9128 & 85.91279 & -85.9128 & 85.91279 & -85.9128 & 85.91279 \\
12 & 87.62928 & -87.6293 & -87.6293 & 87.62928 & -87.6293 & 87.62928 & 87.62928 & -87.6293 \\
13 & 80.79923 & 80.79923 & 80.79923 & -80.7992 & -80.7992 & 80.79923 & 80.79923 & -80.7992 \\
14 & 82.03279 & -82.0328 & 82.03279 & -82.0328 & -82.0328 & 82.03279 & -82.0328 & 82.03279 \\
\hline
\end{tabular}




\begin{tabular}{|c|c|c|c|c|c|c|c|c|}
\hline 15 & 78.91772 & 78.91772 & -78.9177 & -78.9177 & -78.9177 & 78.91772 & -78.9177 & -78.9177 \\
\hline 16 & 79.54887 & -79.5489 & -79.5489 & -79.5489 & -79.5489 & 79.54887 & 79.54887 & 79.54887 \\
\hline 17 & 83.60205 & 83.60205 & 83.60205 & 83.60205 & 83.60205 & -83.602 & 83.60205 & 83.60205 \\
\hline 18 & 85.7452 & -85.7452 & 85.7452 & 85.7452 & 85.7452 & -85.7452 & -85.7452 & -85.7452 \\
\hline 19 & 73.71796 & 73.71796 & -73.718 & 73.71796 & 73.71796 & -73.718 & -73.718 & 73.71796 \\
\hline 20 & 76.22091 & -76.2209 & -76.2209 & 76.22091 & 76.22091 & -76.2209 & 76.22091 & -76.2209 \\
\hline 21 & 70.99306 & 70.99306 & 70.99306 & -70.9931 & 70.99306 & -70.9931 & 70.99306 & -70.9931 \\
\hline 22 & 74.11563 & -74.1156 & 74.11563 & -74.1156 & 74.11563 & -74.1156 & -74.1156 & 74.11563 \\
\hline 23 & 65.5934 & 65.5934 & -65.5934 & -65.5934 & 65.5934 & -65.5934 & -65.5934 & -65.5934 \\
\hline 24 & 68.79341 & -68.7934 & -68.7934 & -68.7934 & 68.79341 & -68.7934 & 68.79341 & 68.79341 \\
\hline 25 & 91.42334 & 91.42334 & 91.42334 & 91.42334 & -91.4233 & -91.4233 & 91.42334 & 91.42334 \\
\hline 26 & 92.69899 & -92.699 & 92.69899 & 92.69899 & -92.699 & -92.699 & -92.699 & -92.699 \\
\hline 27 & 84.44523 & 84.44523 & -84.4452 & 84.44523 & -84.4452 & -84.4452 & -84.4452 & 84.44523 \\
\hline 28 & 87.91037 & -87.9104 & -87.9104 & 87.91037 & -87.9104 & -87.9104 & 87.91037 & -87.9104 \\
\hline 29 & 81.41817 & 81.41817 & 81.41817 & -81.4182 & -81.4182 & -81.4182 & 81.41817 & -81.4182 \\
\hline 30 & 81.72603 & -81.726 & 81.72603 & -81.726 & -81.726 & -81.726 & -81.726 & 81.72603 \\
\hline 31 & 77.64412 & 77.64412 & -77.6441 & -77.6441 & -77.6441 & -77.6441 & -77.6441 & -77.6441 \\
\hline 32 & 79.86298 & -79.863 & -79.863 & -79.863 & -79.863 & -79.863 & 79.86298 & 79.86298 \\
\hline 33 & 65.16427 & -154.987 & 0 & 0 & 0 & 0 & 0 & 0 \\
\hline 34 & 47.34202 & 112.5987 & 0 & 0 & 0 & 0 & 0 & 0 \\
\hline 35 & 84.75804 & 0 & -201.589 & 0 & 0 & 0 & 0 & 0 \\
\hline 36 & 93.10076 & 0 & 221.4318 & 0 & 0 & 0 & 0 & 0 \\
\hline 37 & 51.82756 & 0 & 0 & -123.267 & 0 & 0 & 0 & 0 \\
\hline 38 & 97.12532 & 0 & 0 & 231.0038 & 0 & 0 & 0 & 0 \\
\hline 39 & 100 & 0 & 0 & 0 & -237.841 & 0 & 0 & 0 \\
\hline 40 & 87.49923 & 0 & 0 & 0 & 208.109 & 0 & 0 & 0 \\
\hline 41 & 78.67269 & 0 & 0 & 0 & 0 & -187.116 & 0 & 0 \\
\hline 42 & 93.75402 & 0 & 0 & 0 & 0 & 222.9855 & 0 & 0 \\
\hline 43 & 96.60561 & 0 & 0 & 0 & 0 & 0 & 0 & 0 \\
\hline 44 & 96.06727 & 0 & 0 & 0 & 0 & 0 & 0 & 0 \\
\hline 45 & 97.94627 & 0 & 0 & 0 & 0 & 0 & 0 & 0 \\
\hline 46 & 96.95424 & 0 & 0 & 0 & 0 & 0 & 0 & 0 \\
\hline 47 & 97.46075 & 0 & 0 & 0 & 0 & 0 & 0 & 0 \\
\hline 48 & 96.2487 & 0 & 0 & 0 & 0 & 0 & 0 & 0 \\
\hline 49 & 97.21003 & 0 & 0 & 0 & 0 & 0 & 0 & 0 \\
\hline 50 & 96.95424 & 0 & 0 & 0 & 0 & 0 & 0 & 0 \\
\hline 51 & 95.32283 & 0 & 0 & 0 & 0 & 0 & 0 & 0 \\
\hline 52 & 95.03638 & 0 & 0 & 0 & 0 & 0 & 0 & 0 \\
\hline Sum & 4310.925 & -79.1487 & 107.9892 & 259.8919 & -194.438 & 29.92239 & 0.655258 & -2.41555 \\
\hline
\end{tabular}

Table 4.Continued

\begin{tabular}{rrrrrrrrc}
\hline $\boldsymbol{X}_{\mathbf{1}} \boldsymbol{X}_{\mathbf{4}} \boldsymbol{Y}_{\boldsymbol{b}}$ & $\boldsymbol{X}_{\mathbf{1}} \boldsymbol{X}_{\mathbf{5}} \boldsymbol{Y}_{\boldsymbol{b}}$ & $\boldsymbol{X}_{\mathbf{2}} \boldsymbol{X}_{\mathbf{3}} \boldsymbol{Y}_{\boldsymbol{b}}$ & $\boldsymbol{X}_{\mathbf{2}} \boldsymbol{X}_{\mathbf{4}} \boldsymbol{Y}_{\boldsymbol{b}}$ & $\boldsymbol{X}_{\mathbf{2}} \boldsymbol{X}_{\mathbf{5}} \boldsymbol{Y}_{\boldsymbol{b}}$ & $\boldsymbol{X}_{\mathbf{3}} \boldsymbol{X}_{\mathbf{4}} \boldsymbol{Y}_{\boldsymbol{b}}$ & $\boldsymbol{X}_{\mathbf{3}} \boldsymbol{X}_{\mathbf{5}} \boldsymbol{Y}_{\boldsymbol{b}}$ & $\boldsymbol{X}_{\mathbf{4}} \boldsymbol{X}_{\mathbf{5}} \boldsymbol{Y}_{\boldsymbol{b}}$ & $\boldsymbol{X}_{\mathbf{1}}{ }^{\mathbf{2}} \boldsymbol{Y}_{\boldsymbol{b}}$ \\
\hline 81.23938 & 81.23938 & 81.23938 & 81.23938 & 81.23938 & 81.23938 & 81.23938 & 81.23938 & 81.23938 \\
\hline
\end{tabular}




\begin{tabular}{|c|c|c|c|c|c|c|c|c|}
\hline-84.5462 & -84.5462 & 84.54624 & 84.54624 & 84.54624 & 84.54624 & 84.54624 & 84.54624 & 84.54624 \\
\hline 74.00122 & 74.00122 & -74.0012 & -74.0012 & -74.0012 & 74.00122 & 74.00122 & 74.00122 & 74.00122 \\
\hline-76.6017 & -76.6017 & -76.6017 & -76.6017 & -76.6017 & 76.60166 & 76.60166 & 76.60166 & 76.60166 \\
\hline 69.63233 & 69.63233 & -69.6323 & 69.63233 & 69.63233 & -69.6323 & -69.6323 & 69.63233 & 69.63233 \\
\hline-73.718 & -73.718 & -73.718 & 73.71796 & 73.71796 & -73.718 & -73.718 & 73.71796 & 73.71796 \\
\hline 64.84563 & 64.84563 & 64.84563 & -64.8456 & -64.8456 & -64.8456 & -64.8456 & 64.84563 & 64.84563 \\
\hline-67.2183 & -67.2183 & 67.21829 & -67.2183 & -67.2183 & -67.2183 & -67.2183 & 67.21829 & 67.21829 \\
\hline-90.3715 & 90.37154 & 90.37154 & -90.3715 & 90.37154 & -90.3715 & 90.37154 & -90.3715 & 90.37154 \\
\hline 92.9487 & -92.9487 & 92.9487 & -92.9487 & 92.9487 & -92.9487 & 92.9487 & -92.9487 & 92.9487 \\
\hline-85.9128 & 85.91279 & -85.9128 & 85.91279 & -85.9128 & -85.9128 & 85.91279 & -85.9128 & 85.91279 \\
\hline 87.62928 & -87.6293 & -87.6293 & 87.62928 & -87.6293 & -87.6293 & 87.62928 & -87.6293 & 87.62928 \\
\hline-80.7992 & 80.79923 & -80.7992 & -80.7992 & 80.79923 & 80.79923 & -80.7992 & -80.7992 & 80.79923 \\
\hline 82.03279 & -82.0328 & -82.0328 & -82.0328 & 82.03279 & 82.03279 & -82.0328 & -82.0328 & 82.03279 \\
\hline-78.9177 & 78.91772 & 78.91772 & 78.91772 & -78.9177 & 78.91772 & -78.9177 & -78.9177 & 78.91772 \\
\hline 79.54887 & -79.5489 & 79.54887 & 79.54887 & -79.5489 & 79.54887 & -79.5489 & -79.5489 & 79.54887 \\
\hline 83.60205 & -83.602 & 83.60205 & 83.60205 & -83.602 & 83.60205 & -83.602 & -83.602 & 83.60205 \\
\hline-85.7452 & 85.7452 & 85.7452 & 85.7452 & -85.7452 & 85.7452 & -85.7452 & -85.7452 & 85.7452 \\
\hline 73.71796 & -73.718 & -73.718 & -73.718 & 73.71796 & 73.71796 & -73.718 & -73.718 & 73.71796 \\
\hline-76.2209 & 76.22091 & -76.2209 & -76.2209 & 76.22091 & 76.22091 & -76.2209 & -76.2209 & 76.22091 \\
\hline 70.99306 & -70.9931 & -70.9931 & 70.99306 & -70.9931 & -70.9931 & 70.99306 & -70.9931 & 70.99306 \\
\hline-74.1156 & 74.11563 & -74.1156 & 74.11563 & -74.1156 & -74.1156 & 74.11563 & -74.1156 & 74.11563 \\
\hline 65.5934 & -65.5934 & 65.5934 & -65.5934 & 65.5934 & -65.5934 & 65.5934 & -65.5934 & 65.5934 \\
\hline-68.7934 & 68.79341 & 68.79341 & -68.7934 & 68.79341 & -68.7934 & 68.79341 & -68.7934 & 68.79341 \\
\hline-91.4233 & -91.4233 & 91.42334 & -91.4233 & -91.4233 & -91.4233 & -91.4233 & 91.42334 & 91.42334 \\
\hline 92.69899 & 92.69899 & 92.69899 & -92.699 & -92.699 & -92.699 & -92.699 & 92.69899 & 92.69899 \\
\hline-84.4452 & -84.4452 & -84.4452 & 84.44523 & 84.44523 & -84.4452 & -84.4452 & 84.44523 & 84.44523 \\
\hline 87.91037 & 87.91037 & -87.9104 & 87.91037 & 87.91037 & -87.9104 & -87.9104 & 87.91037 & 87.91037 \\
\hline-81.4182 & -81.4182 & -81.4182 & -81.4182 & -81.4182 & 81.41817 & 81.41817 & 81.41817 & 81.41817 \\
\hline 81.72603 & 81.72603 & -81.726 & -81.726 & -81.726 & 81.72603 & 81.72603 & 81.72603 & 81.72603 \\
\hline-77.6441 & -77.6441 & 77.64412 & 77.64412 & 77.64412 & 77.64412 & 77.64412 & 77.64412 & 77.64412 \\
\hline 79.86298 & 79.86298 & 79.86298 & 79.86298 & 79.86298 & 79.86298 & 79.86298 & 79.86298 & 79.86298 \\
\hline 0 & 0 & 0 & 0 & 0 & 0 & 0 & 0 & 368.6235 \\
\hline 0 & 0 & 0 & 0 & 0 & 0 & 0 & 0 & 267.806 \\
\hline 0 & 0 & 0 & 0 & 0 & 0 & 0 & 0 & 0 \\
\hline 0 & 0 & 0 & 0 & 0 & 0 & 0 & 0 & 0 \\
\hline 0 & 0 & 0 & 0 & 0 & 0 & 0 & 0 & 0 \\
\hline 0 & 0 & 0 & 0 & 0 & 0 & 0 & 0 & 0 \\
\hline 0 & 0 & 0 & 0 & 0 & 0 & 0 & 0 & 0 \\
\hline 0 & 0 & 0 & 0 & 0 & 0 & 0 & 0 & 0 \\
\hline 0 & 0 & 0 & 0 & 0 & 0 & 0 & 0 & 0 \\
\hline 0 & 0 & 0 & 0 & 0 & 0 & 0 & 0 & 0 \\
\hline 0 & 0 & 0 & 0 & 0 & 0 & 0 & 0 & 0 \\
\hline 0 & 0 & 0 & 0 & 0 & 0 & 0 & 0 & 0 \\
\hline
\end{tabular}




\begin{tabular}{lllllllll}
\hline 0 & 0 & 0 & 0 & 0 & 0 & 0 & 0 & 0 \\
0 & 0 & 0 & 0 & 0 & 0 & 0 & 0 & 0 \\
0 & 0 & 0 & 0 & 0 & 0 & 0 & 0 & 0 \\
0 & 0 & 0 & 0 & 0 & 0 & 0 & 0 & 0 \\
0 & 0 & 0 & 0 & 0 & 0 & 0 & 0 & 0 \\
0 & 0 & 0 & 0 & 0 & 0 & 0 & 0 & 0 \\
0 & 0 & 0 & 0 & 0 & 0 & 0 & 0 & 0 \\
0 & 0 & 0 & 0 & 0 & 0 & 0 & 0 & 0 \\
-9.90841 & -0.28775 & 24.12526 & 25.05191 & -6.92138 & 9.374568 & 0.920755 & -8.01056 & 3182.304 \\
\hline
\end{tabular}

Table 4.Continued

\begin{tabular}{|c|c|c|c|c|c|}
\hline$X_{2}^{2} Y_{b}$ & $X_{3}^{2} Y_{b}$ & $X_{4}^{2} Y_{b}$ & $X_{5}^{2} Y_{b}$ & $Y_{m}$ & $\left(Y_{b}-Y_{m}\right)^{2}$ \\
\hline 81.23938 & 81.23938 & 81.23938 & 81.23938 & 87.11434 & 34.51512 \\
\hline 84.54624 & 84.54624 & 84.54624 & 84.54624 & 91.51709 & 48.59274 \\
\hline 74.00122 & 74.00122 & 74.00122 & 74.00122 & 79.44759 & 29.66286 \\
\hline 76.60166 & 76.60166 & 76.60166 & 76.60166 & 83.93212 & 53.7356 \\
\hline 69.63233 & 69.63233 & 69.63233 & 69.63233 & 73.11022 & 12.09568 \\
\hline 73.71796 & 73.71796 & 73.71796 & 73.71796 & 77.21151 & 12.20492 \\
\hline 64.84563 & 64.84563 & 64.84563 & 64.84563 & 68.4543 & 13.02248 \\
\hline 67.21829 & 67.21829 & 67.21829 & 67.21829 & 72.63737 & 29.36644 \\
\hline 90.37154 & 90.37154 & 90.37154 & 90.37154 & 95.0673 & 22.05009 \\
\hline 92.9487 & 92.9487 & 92.9487 & 92.9487 & 98.23348 & 27.92887 \\
\hline 85.91279 & 85.91279 & 85.91279 & 85.91279 & 90.52702 & 21.29116 \\
\hline 87.62928 & 87.62928 & 87.62928 & 87.62928 & 93.77498 & 37.76972 \\
\hline 80.79923 & 80.79923 & 80.79923 & 80.79923 & 82.23312 & 2.056057 \\
\hline 82.03279 & 82.03279 & 82.03279 & 82.03279 & 85.09785 & 9.394581 \\
\hline 78.91772 & 78.91772 & 78.91772 & 78.91772 & 80.70368 & 3.189654 \\
\hline 79.54887 & 79.54887 & 79.54887 & 79.54887 & 83.65018 & 16.82076 \\
\hline 83.60205 & 83.60205 & 83.60205 & 83.60205 & 86.62418 & 9.133279 \\
\hline 85.7452 & 85.7452 & 85.7452 & 85.7452 & 90.99102 & 27.51868 \\
\hline 73.71796 & 73.71796 & 73.71796 & 73.71796 & 78.09364 & 19.14658 \\
\hline 76.22091 & 76.22091 & 76.22091 & 76.22091 & 82.54226 & 39.95944 \\
\hline 70.99306 & 70.99306 & 70.99306 & 70.99306 & 72.73497 & 3.034249 \\
\hline 74.11563 & 74.11563 & 74.11563 & 74.11563 & 76.80035 & 7.20774 \\
\hline 65.5934 & 65.5934 & 65.5934 & 65.5934 & 67.21526 & 2.630444 \\
\hline 68.79341 & 68.79341 & 68.79341 & 68.79341 & 71.36242 & 6.599787 \\
\hline 91.42334 & 91.42334 & 91.42334 & 91.42334 & 93.57742 & 4.640028 \\
\hline 92.69899 & 92.69899 & 92.69899 & 92.69899 & 96.70769 & 16.06966 \\
\hline 84.44523 & 84.44523 & 84.44523 & 84.44523 & 88.17336 & 13.89892 \\
\hline 87.91037 & 87.91037 & 87.91037 & 87.91037 & 91.38541 & 12.07587 \\
\hline 81.41817 & 81.41817 & 81.41817 & 81.41817 & 80.85815 & 0.313622 \\
\hline 81.72603 & 81.72603 & 81.72603 & 81.72603 & 83.68697 & 3.845272 \\
\hline 77.64412 & 77.64412 & 77.64412 & 77.64412 & 78.46492 & 0.673716 \\
\hline
\end{tabular}




\begin{tabular}{|c|c|c|c|c|c|}
\hline 79.86298 & 79.86298 & 79.86298 & 79.86298 & 81.37551 & 2.287774 \\
\hline 0 & 0 & 0 & 0 & 62.20889 & 8.734268 \\
\hline 0 & 0 & 0 & 0 & 53.51183 & 38.0665 \\
\hline 479.4622 & 0 & 0 & 0 & 84.55103 & 0.042851 \\
\hline 526.6556 & 0 & 0 & 0 & 96.41716 & 10.99853 \\
\hline 0 & 293.1799 & 0 & 0 & 61.77557 & 98.963 \\
\hline 0 & 549.4218 & 0 & 0 & 90.33316 & 46.13347 \\
\hline 0 & 0 & 565.6834 & 0 & 105.9792 & 35.75113 \\
\hline 0 & 0 & 494.9686 & 0 & 84.61391 & 8.325074 \\
\hline 0 & 0 & 0 & 445.0384 & 86.12845 & 55.5883 \\
\hline 0 & 0 & 0 & 530.351 & 89.41639 & 18.81502 \\
\hline 0 & 0 & 0 & 0 & 96.43361 & 0.029582 \\
\hline 0 & 0 & 0 & 0 & 96.43361 & 0.134211 \\
\hline 0 & 0 & 0 & 0 & 96.43361 & 2.288123 \\
\hline 0 & 0 & 0 & 0 & 96.43361 & 0.27105 \\
\hline 0 & 0 & 0 & 0 & 96.43361 & 1.055012 \\
\hline 0 & 0 & 0 & 0 & 96.43361 & 0.034194 \\
\hline 0 & 0 & 0 & 0 & 96.43361 & 0.602821 \\
\hline 0 & 0 & 0 & 0 & 96.43361 & 0.27105 \\
\hline 0 & 0 & 0 & 0 & 96.43361 & 1.233846 \\
\hline 0 & 0 & 0 & 0 & 96.43361 & 1.952264 \\
\hline 3551.992 & 3388.476 & 3606.526 & 3521.264 & 4442.577 & 872.0221 \\
\hline
\end{tabular}

Table 4.Continued

\begin{tabular}{ccccccccccc}
\hline $\boldsymbol{\beta}_{\mathbf{1 2}}$ & $\boldsymbol{\beta}_{\mathbf{1 3}}$ & $\boldsymbol{\beta}_{\mathbf{1 4}}$ & $\boldsymbol{\beta}_{\mathbf{1 5}}$ & $\boldsymbol{\beta}_{\mathbf{2 3}}$ & $\boldsymbol{\beta}_{\mathbf{2 4}}$ & $\boldsymbol{\beta}_{\mathbf{2 5}}$ & $\boldsymbol{\beta}_{\mathbf{3 4}}$ & $\boldsymbol{\beta}_{\mathbf{3 5}}$ & $\boldsymbol{\beta}_{\mathbf{4 5}}$ & $\boldsymbol{\beta}$ \\
0.02044 & -0.075 & -0.30 & -0.0089 & 0.752 & 0.781 & -0.215 & 0.292 & 0.028 & -0.24 & 96.433 \\
\hline & & & & & & & & & & \\
\hline $\boldsymbol{\beta}_{\mathbf{1}}$ & $\boldsymbol{\beta}_{\mathbf{2}}$ & $\boldsymbol{\beta}_{\mathbf{3}}$ & $\boldsymbol{\beta}_{\mathbf{4}}$ & $\boldsymbol{\beta}_{\mathbf{5}}$ & $\boldsymbol{\beta}_{\mathbf{1 1}}$ & $\boldsymbol{\beta}_{\mathbf{2 2}}$ & $\boldsymbol{\beta}_{33}$ & $\boldsymbol{\beta}_{\mathbf{4 4}}$ & $\boldsymbol{\beta}_{\mathbf{5 5}}$ \\
-1.828 & 2.494 & 6.003 & -4.491 & 0.691 & -6.818 & -1.051 & -3.602 & -0.201 & -1.5311 \\
\hline
\end{tabular}

The adequately of regression model have been checked with Fisher's $\left(\mathrm{F}_{\mathrm{R}}\right)$ value and tabular value $\left(\mathrm{F}_{\mathrm{T}}\right)$ by following equations:

$$
F_{R}=\frac{S_{A D}^{2}}{S_{Y}^{2}}
$$

For calculation of $\mathrm{S}_{\mathrm{AD}}^{2}$, the expression:

$$
\begin{gathered}
S_{\mathrm{AD}}^{2}=\frac{\mathrm{SS}_{\mathrm{R}}-S S_{\mathrm{E}}}{\mathrm{f}_{\mathrm{AD}}}=\frac{\sum_{1}^{\mathrm{N}}(\mathrm{Yb}-\widehat{\mathrm{Y}} \mathrm{m})^{2}-\left(\mathrm{Y}_{\circ \mathrm{j}}-\overline{\mathrm{Y}}_{\circ}\right)^{2}}{\mathrm{~N}-\left(\mathrm{n}_{\circ}-1\right)-\lambda} \\
\mathrm{S}_{\overline{\mathrm{Y}}}^{2}=\frac{\mathrm{S}_{\mathrm{Y}}^{2}}{\mathrm{~N}} \\
\mathrm{~S}_{\overline{\mathrm{Y}}}^{2}=\frac{\sum_{1}^{\mathrm{n}_{\circ}}\left(\mathrm{Y}_{\circ \mathrm{j}}-\overline{\mathrm{Y}}_{\circ}\right)^{2}}{\mathrm{n}_{\circ}-1}
\end{gathered}
$$


Knowledge of $S_{A D}^{2}$ and $S_{\bar{Y}}^{2}$ facilitates determination of both calculating the value of Fisher's criterion and simultaneously of the tabular value by which we may compare and accept or reject the hypothesis of lack of fit of the regression model.

Where:

$\mathrm{SS}_{\mathrm{E}}$ is sum of squares of reproducibility variance.

$\mathrm{SS}_{\mathrm{R}}$ is residual sum of squares.

$Y_{b}$ is outcome of each trial.

Ym is calculated response value from regression equation.

$\mathrm{Y}_{\mathrm{o}}$ is the outcome of one trial in null point.

$\bar{Y} \circ$ is average of replications in null point.

The rotatability conditions is defined by following relations:

$$
f_{A D}=N-\lambda-\left(n_{\circ}-1\right)
$$

For second order regression models:

$$
\lambda=\frac{(\mathrm{K}+2)(\mathrm{k}+1)}{2}
$$

The value degree of freedom $\left(\mathrm{f}_{\mathrm{E}}\right)$ is calculated by following equation:

$$
\mathrm{f}_{\mathrm{E}}=\mathrm{N}(\mathrm{n}-1)
$$

Table 5. $\left(\mathrm{F}_{\mathrm{R}}\right)$ and $\left(\mathrm{F}_{\mathrm{T}}\right)$ values

\begin{tabular}{ll}
\hline $\mathbf{Y}_{\mathfrak{j}_{j}}$ & $\left(\mathbf{Y}_{\mathbf{o}_{j}}-\overline{\mathbf{Y}}_{\circ}\right)^{\mathbf{2}}$ \\
\hline 96.60561 & 0.0006239 \\
96.06727 & 0.263544 \\
97.94627 & 1.8649653 \\
96.95424 & 0.1395831 \\
97.46075 & 0.774613 \\
96.2487 & 0.1101788 \\
97.21003 & 0.3961424 \\
96.95424 & 0.1395831 \\
95.32283 & 1.5820683 \\
95.03638 & 2.3847118 \\
sum & sum \\
965.8063 & 7.6560138 \\
\hline
\end{tabular}




\begin{tabular}{llllllll}
\hline$\overline{\mathbf{Y}}_{\circ}$ & $\mathbf{S}_{\overline{\mathbf{Y}}}^{\mathbf{2}}$ & $\mathbf{S}_{\mathrm{AD}}^{2}$ & $\mathbf{S}_{\mathbf{Y}}^{2}$ & $\mathrm{~F}_{\mathrm{R}}$ & $\mathbf{f}_{\mathbf{A D}}$ & $\mathbf{f}_{\mathbf{E}}$ & $\mathrm{F}_{\mathrm{T}}$ \\
96.58063 & 0.8506682 & 39.28937 & 44.23475 & 0.888201 & 22 & 468 & 2.31 \\
\hline
\end{tabular}

A tabular value $\mathrm{F}_{\mathrm{T}}$ is obtained for $\mathrm{f}_{\mathrm{AD}}=22$ and $\mathrm{f}_{\mathrm{E}}=52(10-1)=468$ and 1- $\alpha=99 \%$, from Table A.2 in Appendix A (Lazic, 2004).

The regression model is adequate with $99 \%$ confidence because $\mathrm{F}_{\mathrm{R}}<\mathrm{F}_{\mathrm{T}}$ (Fisher's value $<$ tabular value).

A check of significance of regression coefficients is brought down to determining their confidence intervals and their comparison to absolute values of regression coefficients. The rule is (Lazic, 2004):

A regression coefficient is statistically significant if its absolute value is higher than the confidence interval.

When estimating the significance of regression coefficients, these equations are used:

$$
\begin{gathered}
S_{\beta^{\circ}}^{2}=\frac{2 \mathrm{~A} \lambda(\mathrm{K}+2)}{\mathrm{N}} S_{\overline{\mathrm{Y}}}^{2} \\
\mathrm{~S}_{\beta \mathrm{i}}^{2}=\frac{\mathrm{S}_{\overline{\mathrm{Y}}}^{2}}{\mathrm{~N}-\mathrm{n} \circ} \\
\mathrm{S}_{\beta_{\mathrm{ij}}}^{2}=\frac{\mathrm{C}^{2}}{\mathrm{~N}} S_{\overline{\mathrm{Y}}}^{2} \\
\mathrm{~S}_{\beta \mathrm{ii}}^{2}=\frac{\mathrm{AC}^{2}[(\mathrm{~K}+2) \lambda-(\mathrm{K}-2)]}{\mathrm{N}} S_{\overline{\mathrm{Y}}}^{2} \\
\mathrm{~A}=\frac{1}{2 \lambda[(\mathrm{K}+2) \lambda-\mathrm{K}]} \\
\mathrm{C}=\frac{\mathrm{N}}{\mathrm{N}-\mathrm{n} \circ}
\end{gathered}
$$

Where:

$\mathrm{S}_{\beta^{\circ}}, \mathrm{S}_{\beta_{\mathrm{i}}}, \mathrm{S}_{\beta_{\mathrm{ij}}}$ and $\mathrm{S}_{\beta_{\mathrm{ii}}}$ are variance of regression coefficients which associated error mean squares in determining regression coefficients $\beta_{\circ}, \beta_{\mathrm{i}}, \beta_{\mathrm{ij}}$ and $\mathrm{S}_{\beta_{\mathrm{ii}}}$.

In the case of second-order designs of regression coefficient significances, they are checked by using: 
Table 6. $\Delta \beta_{\circ}, \Delta \beta_{\mathrm{i}}, \Delta \beta_{\mathrm{ij}}$ and $\Delta \beta_{\mathrm{ii}}$ values

\begin{tabular}{llll}
\hline $\mathbf{S}_{\boldsymbol{\beta}^{\circ}}$ & 0.016359 & $\Delta \boldsymbol{\beta}_{\circ}=\mp \mathbf{2} \mathbf{S}_{\boldsymbol{\beta}^{\circ}}$ & $\mp 0.255805$ \\
$\mathbf{S}_{\boldsymbol{\beta}_{\mathrm{i}}}$ & 0.0236297 & $\Delta \beta_{\mathrm{i}}=\mp 2 \mathrm{~S}_{\beta_{\mathrm{i}}}$ & $\mp 0.307439$ \\
$\mathbf{S}_{\boldsymbol{\beta}_{\mathrm{ij}}}$ & 0.0265834 & $\Delta \beta_{\mathrm{ij}}=\mp 2 \mathrm{~S}_{\beta_{\mathrm{ij}}}$ & $\mp 0.326088$ \\
$\mathbf{S}_{\boldsymbol{\beta}_{\mathrm{ii}}}$ & 0.0862951 & $\Delta \beta_{\mathrm{ii}}=\mp 2 \mathrm{~S}_{\beta_{\mathrm{ii}}}$ & $\mp 0.587521$ \\
\hline
\end{tabular}

A check of statistical significance of regression coefficients indicates that regression coefficients $\beta, \beta_{1}, \beta_{23}, \beta_{24}, \beta_{11}, \beta_{22}, \beta_{33}$ and $\beta_{55}$ are statistically significant, while the other coefficients are insignificant.The final form of the second order regression model with $99 \%$ confidence may be given in the form:

$$
\begin{array}{r}
Y_{b}=96.433-1.828 X_{1}+2.494 X_{2}+6.003 X_{3}-4.491 X_{4}+0.691 X_{5}-6.818 X_{1}{ }^{2}- \\
1.051 X_{2}{ }^{2}-3.602 X_{3}{ }^{2}-1.5311 X_{5}^{2}+0.752 X_{2} X_{3}+0.781 X_{2} X_{4}
\end{array}
$$

The same above calculations method are used to determine the models ofFe ${ }^{3+}, \mathrm{Pb}^{2+}$ and $\mathrm{Zn}^{2+}$ in batch process. The regression coefficients and final model equations are show below.

For $\mathrm{Fe}^{3+}$ ions were adsorbed on the RHAC in batch process:

\begin{tabular}{|c|c|c|c|c|c|c|c|c|c|c|}
\hline \multicolumn{11}{|l|}{$\beta_{1}$} \\
\hline \multirow[t]{2}{*}{1.544} & 3.275 & 6.2902 & -4.071 & 0.458 & -5.782 & -0.689 & -2.315 & 0.1423 & \multicolumn{2}{|l|}{-1.383} \\
\hline & & & & & & & & & $\beta_{45}$ & $\beta$ \\
\hline 0.252 & -0.50 & 0.021 & 0.175 & -0.071 & 0.348 & -0.155 & 2.007 & 0.073 & 0.064 & 93.962 \\
\hline$\overline{\mathbf{Y}}$ & & $S_{\bar{Y}}^{2}$ & & $S_{A D}^{2}$ & $S$ & $\frac{2}{Y}$ & $F_{R}$ & $\mathbf{f}_{\mathrm{AD}}$ & $\mathbf{f}_{\mathrm{E}}$ & $\mathrm{F}_{\mathrm{T}}$ \\
\hline 94.067 & & 1.9549847 & & 1.14317 & 101.6 & & 0.306349 & 22 & 468 & 2.31 \\
\hline
\end{tabular}

Table 7. The calculated regression coefficients for $\mathrm{Fe}^{3+}$ ions

A check of statistical significance of regression coefficients indicates that regression coefficients $\beta, \beta_{i}, \beta_{13}, \beta_{34}, \beta_{11}, \beta_{33}$ and $\beta_{55}$ are statistically significant, while the other coefficients are insignificant. The final form of the second order regression model with $99 \%$ confidence may be given in the form:

$$
\begin{gathered}
Y_{b}=93.962+1.544 X_{1}+3.275 X_{2}+6.2902 X_{3}-4.071 X_{4}+0.458 X_{5}-5.782 X_{1}{ }^{2}- \\
2.315 \mathrm{X}_{3}{ }^{2}-1.383 X_{5}{ }^{2}+0.752 X_{1} X_{3}+0.781 X_{3} X_{4}
\end{gathered}
$$


For $\mathrm{Pb}^{2+}$ ions were adsorbed on the RHAC in batch process:

Table 8. The calculated regression coefficients for $\mathrm{Pb}^{2+}$ ions

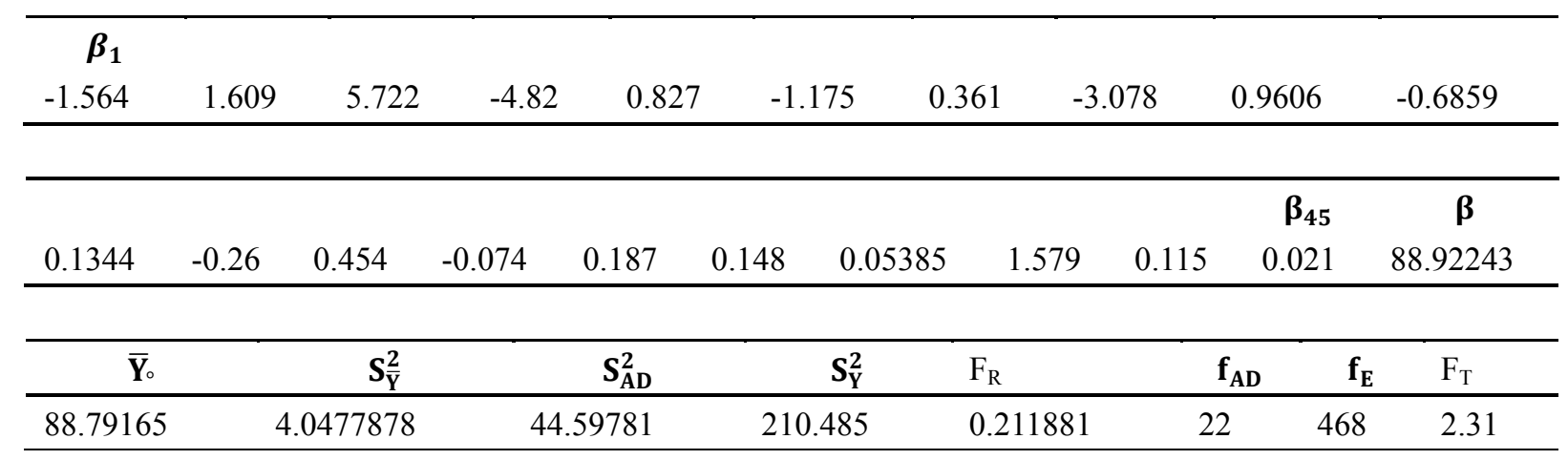

A check of statistical significance of regression coefficients indicates that regression coefficients $\beta, \beta_{i}, \beta_{11}, \beta_{33}$ and $\beta_{34}$ are statistically significant, while the other coefficients are insignificant. The final form of the second order regression model with $99 \%$ confidence may be given in the form:

$$
\begin{gathered}
Y_{b}=88.922-1.564 X_{1}+1.609 X_{2}+5.722 X_{3}-4.82 X_{4}+0.827 X_{5}-1.175 X_{1}^{2}- \\
3.078 X_{3}^{2}+1.579 X_{3} X_{4}
\end{gathered}
$$

For $\mathrm{Zn}^{2+}$ ions were adsorbed on the RHAC in batch process:

Table 9. The calculated regression coefficients for $\mathrm{Zn}^{2+}$ ions

\begin{tabular}{ccccccccccc}
\hline $\begin{array}{c}\boldsymbol{\beta}_{\mathbf{1}} \\
5.467\end{array}$ & 2.961 & 7.053 & -8.78 & 0.424 & -5.396 & -0.35678 & -2.539 & 1.1253 & -1.882 \\
\hline & & & & & & & & & & \\
\hline & & & & & & & & & $\boldsymbol{\beta}_{\mathbf{4 5}}$ & $\boldsymbol{\beta}$ \\
0.3605 & -0.28 & 1.583 & -0.336 & 0.166 & 0.817 & 0.0218 & -1.14 & 0.194 & 0.277 & 73.368 \\
\hline \multicolumn{2}{c}{} & & & & & & & & & \\
\hline$\overline{\mathbf{Y}}_{\circ}$ & $\mathbf{S}_{\mathbf{Y}}^{2}$ & & $\mathbf{S}_{\mathbf{A D}}^{2}$ & $\mathbf{S}_{\mathbf{Y}}^{2}$ & $\mathrm{~F}_{\mathrm{R}}$ & & $\mathbf{f}_{\mathrm{AD}}$ & $\mathbf{f}_{\mathbf{E}}$ & $\mathrm{F}_{\mathrm{T}}$ \\
\hline 73.89135 & 2.9076597 & 57.52014 & 151.1983 & 0.380428 & 22 & 468 & 2.31 \\
\hline
\end{tabular}

A check of statistical significance of regression coefficients indicates that regression coefficients $\beta, \beta_{i}, \beta_{14}, \beta_{24}, \beta_{34}, \beta_{11}, \beta_{33}, \beta_{44}$ and $\beta_{55}$ are statistically significant, while the other coefficients are insignificant. The final form of the second order regression model with $99 \%$ confidence may be given in the form: 


$$
\mathrm{Y}_{\mathrm{b}}=73.368+5.467 \mathrm{X}_{1}+2.961 \mathrm{X}_{2}+7.053 \mathrm{X}_{3}-8.78 \mathrm{X}_{4}+0.424 \mathrm{X}_{5}-5.396 \mathrm{X}_{1}{ }^{2}-
$$

$$
2.539 \mathrm{X}_{3}^{2}+1.1253 \mathrm{X}_{4}{ }^{2}-1.882 \mathrm{X}_{5}{ }^{2}+1.583 \mathrm{X}_{1} \mathrm{X}_{4}+0.817 \mathrm{X}_{2} \mathrm{X}_{4}-1.14 \mathrm{X}_{3} \mathrm{X}_{5}
$$

\subsection{Modeling Using Minitab Software}

Minitab is a statistical software, it was developed by Minitab Inc. (USA).Minitab16.1.0, was used in this study to determine the models of adsorption of heavy metal ions on RHAC.

The calculations and results of models which were determined by this program are listed in Appendix (B), clarification and explanation of the tables and calculations are shown below.

The coefficients table is listed the estimated coefficients for the variables.

Regression examines the relationship between a response and variables. In order to determine whether or not the observed relationship between the response and variables is statistically significant, need to:

Identify the coefficient p-values: the coefficient value for P (p-value) tells whether or not the association between the response and variables is statistically significant. Compare the coefficient $p$-values to $\alpha$-level: if the $p$-value is smaller than the $\alpha$-level, the association is statistically significantly.

$\mathrm{P}$ regression was used to test the hypothesis that all the coefficients in the model are zero. A smaller $p$-value than a pre-selected selected $\alpha$-level implies that at least one coefficient in the model is not zero.

P lack of fit was used to test whether the model fits the data well. A smaller p-value than $\alpha$-level indicates that might need to consider higher order terms of existing predictors, or additional predictors, to get a better fit of the data.

A list of the standard errors for the estimated constant and the estimated coefficient. A standard error for an estimated coefficient measures the precision of the estimate. The smaller the standard error, the more precise the estimate.

$\mathrm{S}$ is measured in the units of the response variable and represents the standard distance data values fall from the regression line. For a given study, the betterequation that predicts the response, the lower $\mathrm{S}$ is.

Minitab displays the coefficients in uncoded units in addition to coded units. For each term in the model, there is a coefficient. Use these coefficients to construct an equation representing the relationship between the response and the factors.

To use this equation, put in the uncoded (actual) factor values and calculate the variables response. Because these coefficients are estimated using uncoded units, putting coded factor values into this equation would produce incorrect predictions about yield.

Note: the above clarification and explanationwere quoted from the help of program.

The final model equations were calculated by this program are shown below: 
For $\mathrm{Cu}^{2+}$ ions were adsorbed on the RHAC in batch process:

$$
\begin{aligned}
Y_{b}=96.3394-1.8273 X_{1}+ & 2.4932 X_{2}+6.0 X_{3}-4.489 X_{4}-7.41 X_{1}{ }^{2}-1.6345 X_{2}{ }^{2}- \\
& 4.189 X_{3}{ }^{2}-2.1146 X_{5}{ }^{2}
\end{aligned}
$$

For $\mathrm{Fe}^{3+}$ ions were adsorbed on the RHAC in batch process:

$$
\begin{gathered}
Y_{b}=93.868+1.543 X_{1}+3.27 X_{2}+6.28 X_{3}-4.069 X_{4}-6.38 X_{1}{ }^{2}-1.278 X_{2}{ }^{2}- \\
2.906 X_{3}{ }^{2}-1.973 X_{5}{ }^{2}+2.01 X_{3} X_{4}
\end{gathered}
$$

For $\mathrm{Pb}^{2+}$ ions were adsorbed on the RHAC in batch process :

$$
\begin{gathered}
\mathrm{Y}_{\mathrm{b}}=88.827-1.56 \mathrm{X}_{1}+1.608 \mathrm{X}_{2}+5.7198 \mathrm{X}_{3}-4.82 \mathrm{X}_{4}-1.778 \mathrm{X}_{1}{ }^{2}-3.684 \mathrm{X}_{2}{ }^{2}- \\
1.288 \mathrm{X}_{5}{ }^{2}
\end{gathered}
$$

For $\mathrm{Zn}^{2+}$ ions were adsorbed on the RHAC in batch process:

$$
\begin{gathered}
Y_{b}=73.295+5.464 X_{1}+2.96 X_{2}+7.049 X_{3}-8.781 X_{4}-5.855 X_{1}{ }^{2}-2.993 X_{3}{ }^{2}- \\
2.3357 X_{5}{ }^{2}
\end{gathered}
$$

\subsection{Validity of Models}

Thevalidity of each equations of model can be tested by the Sum of Squared Errors (SSE \%), the sum of squared errors was determined by following (Tanet al., 2007):

$$
S S E \%=\sqrt{\frac{\sum\left(R P_{\text {exp }}-R P_{c a l}\right)^{2}}{N}}
$$

The lower value of SSE is indicate the better, which indicates that the best model can be chosen.

Table 10. The values of sum of squared errors

\begin{tabular}{lllllllll}
\hline H.M.I. & \multicolumn{2}{c}{$\mathbf{C u}^{2+}$} & \multicolumn{2}{c}{$\mathbf{F e}^{3+}$} & \multicolumn{2}{c}{$\mathbf{P b}^{\mathbf{2 +}}$} & \multicolumn{2}{c}{$\mathbf{Z n}^{2+}$} \\
\hline Models & CCD & Minitab & CCD & Minitab & CCD & Minitab & CCD & Minitab \\
SSE $\%$ & 4.252 & 3.370 & 3.676 & 2.523 & 4.304 & 6.715 & 4.983 & 4.973 \\
\hline
\end{tabular}

Where:

$\mathrm{N}$ is the number of data points. $\mathrm{RP}_{\exp }$ is the values of removal percentage from experimental work. $\mathrm{RP}_{\text {cal }}$ is the values of removal percentage which calculated from models.H.M.I. is heavy metal ions. 


\section{Conclusion}

A mathematical model wasconstructedaccording to Central Composite Design method (CCD) and a software program (minitab16). These models were simulated experimental work for adsorption of $\left(\mathrm{Cu}^{2+}, \mathrm{Fe}^{3+}, \mathrm{Pb}^{2+}\right.$ and $\left.\mathrm{Zn}^{2+}\right)$ in batch adsorption processes using activated carbon produced from rice husk as local raw material which is low cost and available in huge quantities causing a pollutant problem. Final modeling equations were well simulated experimental work with very little deviation by Fisher's testing (1\%), as well as the results of equations derived using (minitab16).

\section{References}

Al-Badran, F. (2003). Chlorinated polyethylene Wax from Industrial Waste: Production, Characterization, Modeling and Kinetic Study. (M.Sc. Thesis). Chemical Engineering Department, College of Engineering, Basrah University.

Alhamed, Y. A., \& Bamufleh, H. S. (2008). Sulfur Removal From Model Diesel Fuel Using Granular Activated Carbon From Dates' Stones Activated by $\mathrm{ZnCl}_{2}$. Journal of Fuel, 88, 87-94. http://dx.doi.org/10.1016/j.fuel.2008.07.019

Al-Jomaa, S. F. (2011). A new local adsorbent for the treatment of an industrial wastewater from toxic metals. (M. Sc. Thesis). Chemical Engineering Department, College of Engineering, Basrah University.

Allen, T. T. (2006). Introduction to Engineering Statistics and Six Sigma. Springer-Verlag London Limited.

Al-tameemi, I. A., Nasser, T., \& Thuraya, M. A. (2012). Removal of pb (II), cd (II) Ions onto dried Conocarpus erectus leaves using batch adsorption and study the adsorption thermodynamics. Journal of Chem. Pharm. Res., 12, 4961-4968.

Ambursa, M. M., Faruk, U. Z., Uba, A., Sahabi, D. M., Atiku, F. A., \& Koko, R. A. (2011). Comparative efficiency of base activated carbon and acid activated carbon for sorption of heavy metals from waste water. Journal of Chem. Pharm. Res., 3(6), 732-741.

Aslan, N., \& Cebeci, Y. (2007). Application of Box-Behnken design and response surface methodology for modeling of some Turkish coals. Journal of Fuel, 86, 90-97. http://dx.doi.org/10.1016/j.fuel.2006.06.010

Badmus, M. A. O., Audu, T. O. K., \& Anyata, B. U. (2007). Removal of Lead Ion from Industrial Wastewaters by Activated Carbon Prepared from Periwinkle Shells. Turkish, Journal Eng. Env. Sci., 31, 251-263.

Belhachemi, M., Rios, R., Addoun, F., Albero, J. S., Escribano, A. S., \& Reinoso, F. R. (2009). Preparation of activated carbon from date pits: Effect of the activation agent and liquid phase oxidation. Journal of Analytical and Applied Pyrolysis, 86, 168-172. http://dx.doi.org/10.1016/j.jaap.2009.05.004

Chen, Y., Zhu, Y., Wang, Z., Li, Y., Wang, L., Lili, Ding, X. G., Ma, Y., \& Guo, Y. (2011). 
Application studies of activated carbon derived from rice husks produced by chemical-thermal process. Advances in Colloid and Interface Science, 163, 39-52. http://dx.doi.org/10.1016/j.cis.2011.01.006

Dada, A. O., Olalekan, A. P., Olatunya, A. M., \& Dada, O. (2012). Langmuir, Freundlich, Temkin and Dubinin-Radushkevich Isotherms Studies of Equilibrium Sorption of $\mathrm{Zn}^{2+}$ Unto Phosphoric Acid Modified Rice Husk. IOSR Journal of Applied Chemistry (IOSR-JAC), 3(1), $38-45$.

Daffalla, S. B., Mukhtar, H., \& Shaharun, M. S. (2012). Effect of Organic and Inorganic Acid Pretreatment on Structural Properties of Rice Husk and Adsorption Mechanism of Phenol. International Journal of Chemical and Environmental Engineering, 3(3), 192-200.

Deosarkar, S. D. (2012). Cu(II) Adsorption from Aqueous Solution by Punicagranatum L. Husk. Journal of Chem. Pharm. Res, 4(6), 3319-3323.

Ghadeer, J. (2009). Study the Kinetic Characterizations of New Photo-Stabilizer(4-Stearoylresorcinol) Production Using Batch Reactor. (M. Sc. Thesis), Chemical Engineering Department, Basrah University.

Gueu, S., Yao, B., Adouby, K., \& Ado, G. (2006). Heavy Metals Removal in Aqueous Solution by Activated Carbons Prepared from Coconut Shell and Seed Shell of the Plam Tree. Journal of Applied Sciences, 6(13), 2789-2793. http://dx.doi.org/10.3923/jas.2006.2789.2793

Hameed, B. H., Ahmad, A. A., \& Aziz, N. (2011). Adsorption of Reactive Dye on Palm-Oil Industry Waste: Equilibrium, Kinetic and Thermodynamic Studies. Journal of Desalination, 247, 551-560. http://dx.doi.org/10.1016/j.desal.2008.08.005

Itodo, A. U., Abdulrahman, F. W., Hassan, L. G., Maigandi, S. A., \& Itodo, H. U. (2010). Intraparticle Diffusion and Intraparticulate Diffusivities of Herbicide on Derived Activated Carbon. Researcher, 2(2), 74-86.

Itodo, H. U., \& Itodo, A. U. (2010). Surface Coverage and Adsorption Study of Dye Uptake by Derived Acid and Base Treated Mango Seed Shells. Journal Chem. Pharm. Res., 2(3), 673-683.

Jaguaribe, E. F., Medeiros, L. L., Barreto, M. C. S., \& Araujo, L. P. (2000). The Performance of Activated Carbons From Sugarcane Bagasse, Babassu, and Coconut Shells in Removing Residual Chlorine. Brazilian Journal of Chemical Engineering, 22(1), 41-47. http://dx.doi.org/10.1590/S0104-66322005000100005

John, W. M. P. (1998). Statistical Design and Analysis of Experiments. Society for Industrial and Applied Mathematics. http://dx.doi.org/10.1137/1.9781611971149

Juan, F. G., Encinar, J. M., Carmen, M., Sabio, E., Ramiro, A., Canito, J., \& Ganan, J. (2005). Preparation of activated carbons from used tyres by gasification with steam and carbon dioxide: $\quad$ Applied Surface $\quad$ Science, $\quad 252, \quad$ 5999-6004. http://dx.doi.org/10.1016/j.apsusc.2005.11.029 
Kannan, \& Veemaraj. (2009). Removal of Lead (II) Ions by Adsorption onto Bamboo Dust and Commercial Activated Carbons-A Comparative Study. Journal of Chemistry, 6(2), 247-256.

Lazic, Z. R. (2004). Design of Experiments in Chemical Engineering. Wiley-VCH, Germany. http://dx.doi.org/10.1002/3527604162

Mohammadi, S. Z., Karimi, M. A., Afzali, D., \& Mansouri, F. (2010). Removal of Pb(II) from aqueous solutions using activated carbon from Sea-buckthorn stones by chemical activation. Journal of Desalination, 262, 86-93. http://dx.doi.org/10.1016/j.desal.2010.05.048

Mui, E. L. K., Cheung, W. H., Valix, M., \& McKay, G. (2010). Mesoporous activated carbon from waste tyre rubber for dye removal from effluents. journal of Microporous and Mesoporous Materials, 130, 287-294. http://dx.doi.org/10.1016/j.micromeso.2009.11.022

Nwabanne, J. T., \& Igbokwe, P. K. (2012). Adsorption Performance of Packed Bed Column for the removal of Lead (II) using oil Palm Fibre. International Journal of Applied Science and Technology, 2(5), 106-115.

Philip, C. A., \& Girgis, B. S. (1996). Adsorption Characteristics of Microporous Carbons from Apricot Stones Activated by Phosphoric Acid. Journal of Chemical Technology and Biotechnology, 67(3), 248-254. http://dx.doi.org/10.1002/(SICI)1097-4660(199611)67:3<248::AID-JCTB557>3.0.CO;2-1

Prabakaran, R., \& Arivoli, S. (2012). Equilibrium Isotherm, Kinetic and Thermodynamic studies of Rhodamine B Adsorption using Thespesiapopulnea Bark. Journal of Chem. Pharm. Res., 4(10), 4550-4557.

Rosa, G. D., Reynel-Avila, H. E., Bonilla-Petriciolet, A., Cano-Rodriguez, I., Velasco-Santos, C., \& Martinez-Hernandez, A. L. (2008). Recycling Poultry Feathers for Pb Removal from Wastewater: Kinetic and Equilibrium Studies. World Academy of Science, Engineering and Technology, 47, 394-402.

Satya, S. P. M., Ahmed, J., \& Krishnaiah, K. (1997). Production of Activated Carbon from Coconut Shell Char in a Fluidized Bed Reactor. Industrial and Engineering Chemistry Research Journal, 36(9), 3625-3630. http://dx.doi.org/10.1021/ie970190v

Shanthi, T., \& Selvarajan, V. M. (2012). Removal of Cu (II) Ions from Aqueous Solution by Carbon Prepared from Henna Leaves, Pungam Bark and CAC. Journal Chem. Pharm. Res., 4(9), 4296-4306.

Song, X., Zhang, Y., Yan, C., Jiang, W., \& Chang, C. (2012). The Langmuir monolayer adsorption model of organic matter into effective pores in activated carbon. Journal of Colloid and Interface Science, 389, 213-219. http://dx.doi.org/10.1016/j.jcis.2012.08.060

Souag, R., Touaibia, D., Benayada, B., \& Boucenna, A. (2009). Adsorption of Heavy Metals $(\mathrm{Cd}, \mathrm{Zn}$ and $\mathrm{Pb})$ from Water UsingKeratin Powder Prepared from Algerien Sheep Hoofs. European Journal of Scientific Research, 35(3), 416-425. 
Surchi, K. M. S. (2011). Agricultural Wastes as Low Cost Adsorbents for Pb Removal: Kinetics, Equilibrium and Thermodynamics. International Journal of Chemistry, 3(3), 103-112. http://dx.doi.org/10.5539/ijc.v3n3p103

Tan, I. A. W., Ahmad, A. L., \& Hameed, B. H. (2007). Adsorption of basic dye using activated carbon prepared from oil palm shell: batch and fixed bed studies. Journal of Desalination, 225, 13-28. http://dx.doi.org/10.1016/j.desal.2007.07.005

Tan, I. A. W., Ahmad, A. L., \& Hameed, B. H. (2007). Preparation of activated carbon from coconut husk: Optimization study on removal of 2,4,6-trichlorophenol using response surface $\begin{array}{lllll}\text { methodology. Journal of Hazardous } & \text { Materials, } & 153, & \text { 709-717. }\end{array}$ http://dx.doi.org/10.1016/j.jhazmat.2007.09.014

Teng, H., Lin, Y., \& Hsu, L. (2000). Production of Activated Carbons from Pyrolysis of Waste Tires Impregnated with Potassium Hydroxide. Journal of the Air \& Waste Management Association, 50, 1940-1946. http://dx.doi.org/10.1080/10473289.2000.10464221

\section{Appendix}

Appendix A.1. The experimentalresult of adsorption of heavy metalions on the RHAC

\begin{tabular}{|c|c|c|c|c|c|c|c|c|c|}
\hline No. & PH & $\mathbf{T} \dot{\mathrm{C}}$ & Dose & Conc. & rpm & $C u^{2+}$ R.P. $\%$ & $F e^{3+}$ R.P. $\%$ & $P b^{2+}$ R.P. $\%$ & $Z n^{2+}$ R.P. $\%$ \\
\hline 1 & 9 & 50 & 4 & 50 & 750 & 81.23938 & 88.10662 & 83.82919 & 67.9962 \\
\hline 2 & 5 & 50 & 4 & 50 & 750 & 84.54624 & 85.33153 & 85.56493 & 52.9737 \\
\hline 3 & 9 & 22 & 4 & 50 & 750 & 74.00122 & 81.08255 & 79.39755 & 56.61451 \\
\hline 4 & 5 & 22 & 4 & 50 & 750 & 76.60166 & 79.10268 & 81.62985 & 42.66601 \\
\hline 5 & 9 & 50 & 2 & 50 & 750 & 69.63233 & 74.32959 & 72.50283 & 56.61451 \\
\hline 6 & 5 & 50 & 2 & 50 & 750 & 73.71796 & 71.47762 & 74.83404 & 42.66601 \\
\hline 7 & 9 & 22 & 2 & 50 & 750 & 64.84563 & 64.78792 & 68.94422 & 49.15597 \\
\hline 8 & 5 & 22 & 2 & 50 & 750 & 67.21829 & 63.03204 & 70.13866 & 38.46659 \\
\hline 9 & 9 & 50 & 4 & 21 & 750 & 90.37154 & 93.31052 & 89.2343 & 80.17145 \\
\hline 10 & 5 & 50 & 4 & 21 & 750 & 92.9487 & 91.29079 & 93.56186 & 73.17979 \\
\hline 11 & 9 & 22 & 4 & 21 & 750 & 85.91279 & 84.86117 & 84.87837 & 76.66654 \\
\hline 12 & 5 & 22 & 4 & 21 & 750 & 87.62928 & 83.32352 & 91.40162 & 70.96144 \\
\hline 13 & 9 & 50 & 2 & 21 & 750 & 80.79923 & 86.42614 & 84.87837 & 70.3359 \\
\hline 14 & 5 & 50 & 2 & 21 & 750 & 82.03279 & 81.81263 & 87.05988 & 61.41432 \\
\hline 15 & 9 & 22 & 2 & 21 & 750 & 78.91772 & 80.32793 & 81.59281 & 64.78172 \\
\hline 16 & 5 & 22 & 2 & 21 & 750 & 79.54887 & 76.02539 & 85.97001 & 54.57449 \\
\hline 17 & 9 & 50 & 4 & 50 & 350 & 83.60205 & 88.35708 & 84.04731 & 68.70761 \\
\hline 18 & 5 & 50 & 4 & 50 & 350 & 85.7452 & 85.58079 & 85.13297 & 50.95187 \\
\hline 19 & 9 & 22 & 4 & 50 & 350 & 73.71796 & 80.78995 & 79.1725 & 57.98543 \\
\hline 20 & 5 & 22 & 4 & 50 & 350 & 76.22091 & 78.45418 & 82.07236 & 41.31281 \\
\hline 21 & 9 & 50 & 2 & 50 & 350 & 70.99306 & 74.90697 & 71.32487 & 58.76964 \\
\hline 22 & 5 & 50 & 2 & 50 & 350 & 74.11563 & 72.93589 & 74.6024 & 41.76894 \\
\hline 23 & 9 & 22 & 2 & 50 & 350 & 65.5934 & 64.54013 & 69.90043 & 50.60096 \\
\hline 24 & 5 & 22 & 2 & 50 & 350 & 68.79341 & 61.61917 & 71.56112 & 39.4365 \\
\hline 25 & 9 & 50 & 4 & 21 & 350 & 91.42334 & 93.48079 & 89.12575 & 80.4263 \\
\hline 26 & 5 & 50 & 4 & 21 & 350 & 92.69899 & 91.29079 & 93.56186 & 75.56583 \\
\hline 27 & 9 & 22 & 4 & 21 & 350 & 84.44523 & 81.81263 & 85.53357 & 77.66965 \\
\hline 28 & 5 & 22 & 4 & 21 & 350 & 87.91037 & 86.42614 & 90.31885 & 72.31815 \\
\hline 29 & 9 & 50 & 2 & 21 & 350 & 81.41817 & 86.42614 & 85.97001 & 73.86935 \\
\hline 30 & 5 & 50 & 2 & 21 & 350 & 81.72603 & 82.56477 & 88.14798 & 61.76971 \\
\hline
\end{tabular}




\begin{tabular}{llllllllll}
\hline $\mathbf{3 1}$ & 9 & 22 & 2 & 21 & 350 & 77.64412 & 81.06704 & 82.68977 & 66.5229 \\
$\mathbf{3 2}$ & 5 & 22 & 2 & 21 & 350 & 79.86298 & 76.30535 & 84.87837 & 55.21746 \\
$\mathbf{3 3}$ & 2.243 & 36 & 3 & 35.5 & 550 & 65.16427 & 53.23355 & 82.7634 & 33.13241 \\
$\mathbf{3 4}$ & 11.76 & 36 & 3 & 35.5 & 550 & 47.34202 & 65.34776 & 74.23148 & 56.28086 \\
$\mathbf{3 5}$ & 7 & 2.702 & 3 & 35.5 & 550 & 84.75804 & 84.42365 & 83.76516 & 67.8024 \\
$\mathbf{3 6}$ & 7 & 69.3 & 3 & 35.5 & 550 & 93.10076 & 91.87511 & 90.64515 & 78.72574 \\
$\mathbf{3 7}$ & 7 & 36 & 0.6216 & 35.5 & 550 & 51.82756 & 58.27698 & 41.58728 & 30.38296 \\
$\mathbf{3 8}$ & 7 & 36 & 5.3784 & 35.5 & 550 & 97.12532 & 99.60192 & 93.83991 & 91.40932 \\
$\mathbf{3 9}$ & 7 & 36 & 3 & 1.013 & 550 & 100 & 100 & 100 & 98.8141 \\
$\mathbf{4 0}$ & 7 & 36 & 3 & 69.99 & 550 & 87.49923 & 85.7259 & 81.19688 & 64.50927 \\
$\mathbf{4 1}$ & 7 & 36 & 3 & 35.5 & 74.32 & 78.67269 & 79.63733 & 73.18559 & 57.88295 \\
$\mathbf{4 2}$ & 7 & 36 & 3 & 35.5 & 1026 & 93.75402 & 88.79539 & 89.35254 & 71.35046 \\
$\mathbf{4 3}$ & 7 & 36 & 3 & 35.5 & 550 & 96.60561 & 95.66103 & 89.35254 & 73.14867 \\
$\mathbf{4 4}$ & 7 & 36 & 3 & 35.5 & 550 & 96.06727 & 95.66103 & 90.96699 & 74.06098 \\
$\mathbf{4 5}$ & 7 & 36 & 3 & 35.5 & 550 & 97.94627 & 93.81068 & 89.9999 & 70.46211 \\
$\mathbf{4 6}$ & 7 & 36 & 3 & 35.5 & 550 & 96.95424 & 95.98851 & 88.05152 & 73.14867 \\
$\mathbf{4 7}$ & 7 & 36 & 3 & 35.5 & 550 & 97.46075 & 91.87511 & 91.28831 & 75.91122 \\
$\mathbf{4 8}$ & 7 & 36 & 3 & 35.5 & 550 & 96.2487 & 92.67749 & 88.05152 & 75.91122 \\
$\mathbf{4 9}$ & 7 & 36 & 3 & 35.5 & 550 & 97.21003 & 93.52026 & 84.43038 & 74.98215 \\
$\mathbf{5 0}$ & 7 & 36 & 3 & 35.5 & 550 & 96.95424 & 94.71204 & 89.9999 & 72.24541 \\
$\mathbf{5 1}$ & 7 & 36 & 3 & 35.5 & 550 & 95.32283 & 92.95377 & 87.39786 & 74.98215 \\
$\mathbf{5 2}$ & 7 & 36 & 3 & 35.5 & 550 & 95.03638 & 93.81068 & 88.37756 & 74.06098 \\
\hline
\end{tabular}

AppendixA.2. $\mathrm{F}_{\mathrm{T}}$ Values

\begin{tabular}{|c|c|c|c|c|c|c|c|c|c|c|c|c|c|c|c|c|c|c|c|c|c|}
\hline \multirow[t]{2}{*}{$\bar{\alpha}$} & \multirow[t]{2}{*}{$f_{2}$} & \multicolumn{19}{|c|}{$f_{1}$ (for greater variance) } & \multirow[t]{2}{*}{$f_{2}$} \\
\hline & & 1 & 2 & 3 & 4 & 5 & 6 & 7 & 8 & 0 & 0 & 12 & 15 & 20 & 24 & 30 & 0 & 60 & 20 & $\infty$ & \\
\hline $\begin{array}{l}0.1 \\
0.05 \\
0.01\end{array}$ & 19 & $\begin{array}{l}2.99 \\
4.38 \\
8.18\end{array}$ & $\begin{array}{l}2.61 \\
3.52 \\
5.93\end{array}$ & $\begin{array}{l}3.13 \\
5.01\end{array}$ & $\begin{array}{l}2.27 \\
2.90 \\
4.50\end{array}$ & $\begin{array}{l}2.18 \\
2.74 \\
4.17\end{array}$ & $\begin{array}{l}2.11 \\
2.63 \\
3.94\end{array}$ & $\begin{array}{l}2.06 \\
2.54 \\
3.77\end{array}$ & $\begin{array}{l}2.02 \\
2.43 \\
3.63\end{array}$ & $\begin{array}{l}.98 \\
.42 \\
52\end{array}$ & & & & & & $\begin{array}{l}1,76 \\
2.07 \\
2.84\end{array}$ & $\begin{array}{l}1.73 \\
203 \\
276\end{array}$ & & $\begin{array}{l}1.67 \\
1.93 \\
2.58\end{array}$ & $\begin{array}{l}1.63 \\
1.83 \\
2.49\end{array}$ & 19 \\
\hline $\begin{array}{l}0.1 \\
0.05 \\
0.01\end{array}$ & 20 & $\begin{array}{l}4.35 \\
3.10\end{array}$ & $\begin{array}{l}3.49 \\
5.85\end{array}$ & & & & & & & & & & & & & & & & & & 20 \\
\hline $\begin{array}{l}0.1 \\
0.05 \\
0.01\end{array}$ & 21 & 3.02 & $\begin{array}{l}3.47 \\
5.78\end{array}$ & & $\begin{array}{l}2.23 \\
2.84 \\
4.37\end{array}$ & & $\begin{array}{l}2.08 \\
2.57 \\
3.81\end{array}$ & & & $\begin{array}{l}1.95 \\
2.37 \\
3.40\end{array}$ & & & & & & & $\begin{array}{l}1.69 \\
1.96 \\
2.64\end{array}$ & & & & 1 \\
\hline $\begin{array}{l}0.1 \\
0.05 \\
0.01\end{array}$ & 22 & $\begin{array}{l}4.30 \\
7.95\end{array}$ & $\begin{array}{l}2.56 \\
3.44 \\
5.72\end{array}$ & & $\begin{array}{l}2.82 \\
4.31\end{array}$ & & & & & & & & & & & & $\begin{array}{l}1.67 \\
1.94 \\
2.58\end{array}$ & & & $\begin{array}{l}1.57 \\
1.73 \\
2.31\end{array}$ & 22 \\
\hline $\begin{array}{l}0.1 \\
0.05 \\
0.01\end{array}$ & 23 & $\begin{array}{l}2.94 \\
4.28 \\
7.88\end{array}$ & $\begin{array}{l}2.55 \\
3.42 \\
5.66\end{array}$ & & $\begin{array}{l}2.21 \\
2.80 \\
4.26\end{array}$ & $\begin{array}{l}2.11 \\
2.64 \\
3.94\end{array}$ & $\begin{array}{l}2.05 \\
2.53 \\
3.71\end{array}$ & & & $\begin{array}{l}1.92 \\
2.32 \\
3.30\end{array}$ & & & & & & & $\begin{array}{l}1.66 \\
1.91 \\
2.54\end{array}$ & & & & 6 \\
\hline $\begin{array}{l}0.05 \\
0.01\end{array}$ & 24 & $\begin{array}{l}4.26 \\
7.82\end{array}$ & $\begin{array}{l}2.54 \\
3.40 \\
5.61\end{array}$ & $\begin{array}{l}3.01 \\
4.72\end{array}$ & $\begin{array}{l}2.19 \\
2.78 \\
4.22\end{array}$ & $\begin{array}{l}2.10 \\
262 \\
3.90\end{array}$ & $\begin{array}{l}2.04 \\
2.51 \\
3.67\end{array}$ & & $\begin{array}{l}1.94 \\
2.35 \\
3.35\end{array}$ & $\begin{array}{l}1.91 \\
2.30 \\
3.26\end{array}$ & & & $\begin{array}{l}2.11 \\
2.89\end{array}$ & & & $\begin{array}{l}1.67 \\
1.94 \\
2.58\end{array}$ & $\begin{array}{l}1.64 \\
1.89 \\
2.49\end{array}$ & $\begin{array}{l}1.51 \\
1.34 \\
2.40\end{array}$ & $\begin{array}{l}1.57 \\
1.79 \\
2.31\end{array}$ & $\begin{array}{l}1.53 \\
1.73 \\
2.21\end{array}$ & 24 \\
\hline $\begin{array}{l}0.1 \\
0.05 \\
0.01\end{array}$ & 25 & $\begin{array}{l}2.92 \\
4.24 \\
7.77\end{array}$ & $\begin{array}{l}2.53 \\
3.39 \\
5.57\end{array}$ & $\begin{array}{l}2.32 \\
2.99 \\
4.68\end{array}$ & $\begin{array}{l}2.18 \\
2.76 \\
4.18\end{array}$ & $\begin{array}{l}209 \\
260 \\
3.86\end{array}$ & $\begin{array}{l}2.02 \\
2.49 \\
3.63\end{array}$ & $\begin{array}{l}1.97 \\
2.40 \\
3.46\end{array}$ & $\begin{array}{l}1.93 \\
2.34 \\
3.32\end{array}$ & $\begin{array}{l}1.39 \\
2.28 \\
3.22\end{array}$ & & $\begin{array}{l}1.82 \\
2.15 \\
2.99\end{array}$ & $\begin{array}{l}1.77 \\
2.09 \\
2.85\end{array}$ & $\begin{array}{l}1.72 \\
2.01 \\
2.70\end{array}$ & $\begin{array}{l}1.69 \\
1.96 \\
2.62\end{array}$ & $\begin{array}{l}1.66 \\
1.92 \\
2.54\end{array}$ & $\begin{array}{l}1.63 \\
1.87 \\
2.45\end{array}$ & $\begin{array}{l}1.59 \\
1.52 \\
2.36\end{array}$ & $\begin{array}{l}1.56 \\
1.77 \\
2.27\end{array}$ & $\begin{array}{l}1.52 \\
1.71 \\
2.17\end{array}$ & 25 \\
\hline $\begin{array}{l}0.1 \\
0.05 \\
0.01\end{array}$ & 26 & $\begin{array}{l}2.91 \\
4.23 \\
7.72\end{array}$ & $\begin{array}{l}2.52 \\
3.37 \\
5.53\end{array}$ & $\begin{array}{l}2.31 \\
2.98 \\
4.64\end{array}$ & $\begin{array}{l}2.17 \\
2.74 \\
4.14\end{array}$ & $\begin{array}{l}208 \\
2.59 \\
3.82\end{array}$ & $\begin{array}{l}2.01 \\
2.47 \\
3.59\end{array}$ & $\begin{array}{l}1.96 \\
2.39 \\
3.42\end{array}$ & $\begin{array}{l}1.92 \\
2.32 \\
3.29\end{array}$ & $\begin{array}{l}1.38 \\
2.27 \\
3.18\end{array}$ & $\begin{array}{l}1.86 \\
2.22 \\
3.69\end{array}$ & $\begin{array}{l}1.81 \\
2.15 \\
2.96\end{array}$ & $\begin{array}{l}1.76 \\
2.07 \\
2.82\end{array}$ & $\begin{array}{l}1.71 \\
1.99 \\
2.66\end{array}$ & $\begin{array}{l}1.68 \\
1.95 \\
2.58\end{array}$ & $\begin{array}{l}1.65 \\
1.90 \\
2.50\end{array}$ & $\begin{array}{l}1.61 \\
1.85 \\
2.42\end{array}$ & $\begin{array}{l}1.58 \\
1.30 \\
2.33\end{array}$ & $\begin{array}{l}1.54 \\
1.75 \\
2.23\end{array}$ & $\begin{array}{l}1.50 \\
1.69 \\
2.13\end{array}$ & 26 \\
\hline
\end{tabular}


AppendixB. The calculation and results of models were determined by Minitab program

\section{Central Composite Design}

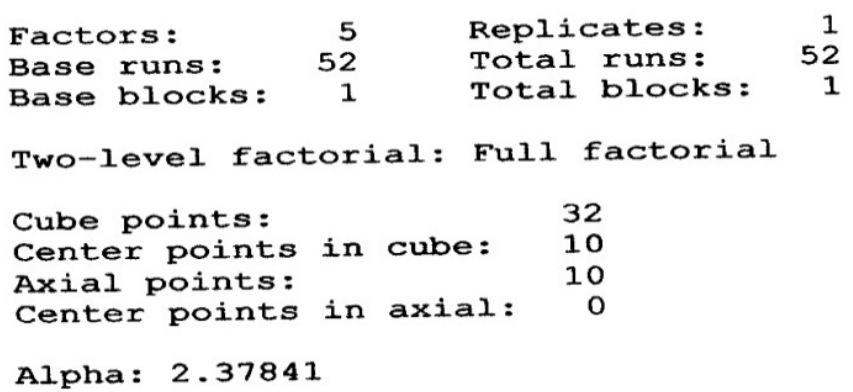

\begin{tabular}{|c|c|c|}
\hline Run & $\mathrm{BIk}$ & A \\
\hline 1 & 1 & -1.00000 \\
\hline 2 & 1 & 1.00000 \\
\hline 3 & 1 & -1.00000 \\
\hline 4 & 1 & 1.00000 \\
\hline 5 & 1 & -1.00000 \\
\hline 6 & 1 & 1.00000 \\
\hline 7 & 1 & -1.00000 \\
\hline 8 & 1 & 1.00000 \\
\hline 9 & 1 & -1.00000 \\
\hline 10 & 1 & 1.00000 \\
\hline 11 & 1 & -1.00000 \\
\hline 12 & 1 & 1.00000 \\
\hline 13 & 1 & -1.00000 \\
\hline 14 & 1 & 1.00000 \\
\hline 15 & 1 & -1.00000 \\
\hline 16 & 1 & 1.00000 \\
\hline 17 & 1 & -1.00000 \\
\hline 18 & 1 & 1.00000 \\
\hline 19 & 1 & -1.00000 \\
\hline 20 & 1 & 1.00000 \\
\hline 21 & 1 & -1.00000 \\
\hline 22 & 1 & 1.00000 \\
\hline 23 & 1 & -1.00000 \\
\hline 24 & 1 & 1.00000 \\
\hline 25 & 1 & -1.00000 \\
\hline 26 & 1 & 1.00000 \\
\hline 27 & 1 & -1.00000 \\
\hline 28 & 1 & 1.00000 \\
\hline 29 & 1 & -1.00000 \\
\hline 30 & 1 & 1.00000 \\
\hline 31 & 1 & -1.00000 \\
\hline 32 & 1 & 1.00000 \\
\hline 33 & 1 & -2.37841 \\
\hline 34 & 1 & 2.37841 \\
\hline 35 & 1 & 0.00000 \\
\hline 36 & 1 & 0.00000 \\
\hline 37 & 1 & 0.00000 \\
\hline 38 & 1 & 0.00000 \\
\hline 39 & 1 & 0.00000 \\
\hline 40 & 1 & 0.00000 \\
\hline 41 & 1 & 0.00000 \\
\hline 42 & 1 & 0.00000 \\
\hline 43 & 1 & 0.00000 \\
\hline 44 & 1 & 0.00000 \\
\hline 45 & 1 & 0.00000 \\
\hline 46 & 1 & 0.00000 \\
\hline 47 & 1 & 0.00000 \\
\hline 48 & 1 & 0.00000 \\
\hline 49 & 1 & 0.00000 \\
\hline 50 & 1 & 0.00000 \\
\hline 51 & 1 & 0.00000 \\
\hline 52 & 1 & 0.00000 \\
\hline
\end{tabular}

$\begin{array}{rr} & D \\ -1.00000 & -1.00000 \\ -1.00000 & -1.00000 \\ -1.00000 & -1.00000 \\ -1.00000 & -1.00000 \\ -1.00000 & -1.00000 \\ -1.00000 & -1.00000 \\ -1.00000 & -1.00000 \\ -1.00000 & -1.00000 \\ 1.00000 & -1.00000 \\ 1.00000 & -1.00000 \\ 1.00000 & -1.00000 \\ 1.00000 & -1.00000 \\ 1.00000 & -1.00000 \\ 1.00000 & -1.00000 \\ 1.00000 & -1.00000 \\ 1.00000 & -1.00000 \\ -1.00000 & 1.00000 \\ -1.00000 & 1.00000 \\ -1.00000 & 1.00000 \\ -1.00000 & 1.00000 \\ -1.00000 & 1.00000 \\ -1.00000 & 1.00000 \\ -1.00000 & 1.00000 \\ -1.00000 & 1.00000 \\ 1.00000 & 1.00000 \\ 1.00000 & 1.00000 \\ 1.00000 & 1.00000 \\ 1.00000 & 1.00000 \\ 1.00000 & 1.00000 \\ 1.00000 & 1.00000 \\ 1.00000 & 1.00000 \\ 1.00000 & 1.00000 \\ 0.00000 & 0.00000 \\ 0.00000 & 0.00000 \\ 0.00000 & 0.00000 \\ 0.00000 & 0.00000 \\ 0.00000 & 0.00000 \\ 0.00000 & 0.00000 \\ -2.37841 & 0.00000 \\ 2.37841 & 0.00000 \\ 0.00000 & -2.37841 \\ 0.00000 & 2.37841 \\ 0.00000 & 0.00000 \\ 0.00000 & 0.00000 \\ 0.00000 & 0.00000 \\ 0.00000 & 0.00000 \\ 0.00000 & 0.00000 \\ 0.00000 & 0.00000 \\ 0.00000 & 0.00000 \\ 0.00000 & 0.00000 \\ 0.00000 & 0.00000 \\ 0.00000 & 0.00000 \\ & \end{array}$




\section{Adsorption of $\mathrm{Cu}^{2+}$ in batch presses}

Response Surface Regression: response versus X1, X2, X3, X4, X5

The analysis was done using coded units.

Estimated Regression Coefficients for response

$\begin{array}{lrrrr}\text { Term } & \text { Coef } & \text { SE Coef } & T & \text { P } \\ \text { Constant } & 96.3394 & 1.2160 & 79.229 & 0.000 \\ \mathrm{X} 1 & -1.8273 & 0.5879 & -3.108 & 0.004 \\ \mathrm{X} 2 & 2.4932 & 0.5879 & 4.241 & 0.000 \\ \mathrm{X} 3 & 6.0002 & 0.5879 & 10.207 & 0.000 \\ \mathrm{X} 4 & -4.4891 & 0.5879 & -7.636 & 0.000 \\ \mathrm{X} 5 & 0.6908 & 0.5879 & 1.175 & 0.249 \\ \mathrm{X} 1 * \mathrm{X} 1 & -7.4109 & 0.5057 & -14.654 & 0.000 \\ \mathrm{X} 2 * \mathrm{X} 2 & -1.6345 & 0.5057 & -3.232 & 0.003 \\ \mathrm{X} 3 * \mathrm{X} 3 & -4.1894 & 0.5057 & -8.284 & 0.000 \\ \mathrm{X} 4 * \mathrm{X} 4 & -0.7824 & 0.5057 & -1.547 & 0.132 \\ \mathrm{X} 5 * \mathrm{X} 5 & -2.1146 & 0.5057 & -4.181 & 0.000 \\ \mathrm{X} 1 * \mathrm{X} 2 & 0.0205 & 0.6839 & 0.030 & 0.976 \\ \mathrm{X} 1 * \mathrm{X} 3 & -0.0755 & 0.6839 & -0.110 & 0.913 \\ \mathrm{X} 1 * \mathrm{X} 4 & -0.3096 & 0.6839 & -0.453 & 0.654 \\ \mathrm{X} 1 * \mathrm{X} 5 & -0.0090 & 0.6839 & -0.013 & 0.990 \\ \mathrm{X} 2 * \mathrm{X} 3 & 0.7539 & 0.6839 & 1.102 & 0.279 \\ \mathrm{X} 2 * \mathrm{X} 4 & 0.7829 & 0.6839 & 1.145 & 0.261 \\ \mathrm{X} 2 * \mathrm{X} 5 & -0.2163 & 0.6839 & -0.316 & 0.754 \\ \mathrm{X} 3 * \mathrm{X} 4 & 0.2930 & 0.6839 & 0.428 & 0.671 \\ \mathrm{X} 3 * \mathrm{X} 5 & 0.0288 & 0.6839 & 0.042 & 0.967 \\ \mathrm{X} 4 * \mathrm{X} 5 & -0.2503 & 0.6839 & -0.366 & 0.717\end{array}$

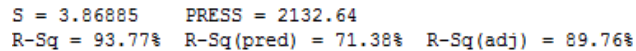

\begin{tabular}{|c|c|c|c|c|c|c|}
\hline burce & DF & Seq SS & Adj SS & Adj MS & $\mathrm{F}$ & 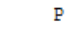 \\
\hline Regression & 20 & 6987.36 & 6987.36 & 349.37 & 23.34 & 0.000 \\
\hline Linear & 5 & 2866.80 & 2866.80 & 573.36 & 38.31 & 0.000 \\
\hline $\mathrm{x} 1$ & 1 & 144.63 & 144.63 & 144.63 & 9.66 & 0.004 \\
\hline $\mathrm{x} 2$ & 1 & 269.24 & 269.24 & 269.24 & 17.99 & 0.000 \\
\hline x3 & 1 & 1559.41 & 1559.41 & 1559.41 & 104.18 & 0.000 \\
\hline $\mathrm{X} 4$ & 1 & 872.84 & 872.84 & 872.84 & 58.31 & 0.000 \\
\hline $\mathrm{x} 5$ & 1 & 20.67 & 20.67 & 20.67 & 1.38 & 0.249 \\
\hline Square & 5 & 4073.22 & 4073.22 & 814.64 & 54.43 & 0.000 \\
\hline $\mathrm{X} 1 * \mathrm{X} 1$ & 1 & 2784.90 & 3214.33 & 3214.33 & 214.75 & 0.000 \\
\hline $\mathrm{X} 2 * \mathrm{X} 2$ & 1 & 74.41 & 156.35 & 156.35 & 10.45 & 0.003 \\
\hline $\mathrm{X} 3 * \mathrm{X} 3$ & 1 & 930.88 & 1027.21 & 1027.21 & 68.63 & 0.000 \\
\hline $\mathrm{X} 4 * \mathrm{X} 4$ & 1 & 21.33 & 35.82 & 35.82 & 2.39 & 0.132 \\
\hline $\mathrm{x} 5 * \mathrm{x} 5$ & 1 & 261.70 & 261.70 & 261.70 & 17.48 & 0.000 \\
\hline Interaction & 10 & 47.34 & 47.34 & 4.73 & 0.32 & 0.971 \\
\hline $\mathrm{X} 1 * \mathrm{X} 2$ & 1 & 0.01 & 0.01 & 0.01 & 0.00 & 0.976 \\
\hline $\mathrm{X} 1 * \mathrm{X} 3$ & 1 & 0.18 & 0.18 & 0.18 & 0.01 & 0.913 \\
\hline $\mathrm{X} 1 * \mathrm{X} 4$ & 1 & 3.07 & 3.07 & 3.07 & 0.20 & 0.654 \\
\hline $\mathrm{X} 1 * \mathrm{X} 5$ & 1 & 0.00 & 0.00 & 0.00 & 0.00 & 0.990 \\
\hline $\mathrm{X} 2 * \mathrm{X} 3$ & 1 & 18.19 & 18.19 & 18.19 & 1.22 & 0.279 \\
\hline $\mathrm{X} 2 * \mathrm{X} 4$ & 1 & 19.61 & 19.61 & 19.61 & 1.31 & 0.261 \\
\hline $\mathrm{x} 2 * \mathrm{x} 5$ & 1 & 1.50 & 1.50 & 1.50 & 0.10 & 0.754 \\
\hline $\mathrm{X} 3 * \mathrm{X} 4$ & 1 & 2.75 & 2.75 & 2.75 & 0.18 & 0.671 \\
\hline$x 3 * x 5$ & 1 & 0.03 & 0.03 & 0.03 & 0.00 & 0.967 \\
\hline $\mathrm{X} 4 * \times 5$ & 1 & 2.01 & 2.01 & 2.01 & 0.13 & 0.717 \\
\hline Residual Error & 31 & 464.01 & 464.01 & 14.97 & & \\
\hline Lack-of-Fit & 22 & 456.35 & 456.35 & 20.74 & 24.38 & 0.000 \\
\hline Pure Error & 9 & 7.66 & 7.66 & 0.85 & & \\
\hline Total & 51 & 7451.37 & & & & \\
\hline
\end{tabular}

Unusual Observations for response

$\begin{array}{rrrrrrr}\text { Obs } & \text { StdOrder } & \text { response } & \text { Fit } & \text { SE Fit } & \text { Residual } & \text { St Resid } \\ 33 & 33 & 65.164 & 58.763 & 2.895 & 6.401 & 2.49 \mathrm{R} \\ 37 & 37 & 51.828 & 58.369 & 2.895 & -6.542 & -2.55 \mathrm{R} \\ 38 & 38 & 97.125 & 86.912 & 2.895 & 10.214 & 3.98 \mathrm{R} \\ 40 & 40 & 87.499 & 81.237 & 2.895 & 6.262 & 2.44 \mathrm{R} \\ 42 & 42 & 93.754 & 86.020 & 2.895 & 7.734 & 3.01 \mathrm{R}\end{array}$

\section{Adsorption of $\mathrm{Fe}^{3+}$ in batch presses}

Response Surface Regression: Response versus X1, X2, X3, X4, X5

The analysis was done using coded units.

Estimated Regression Coefficients for Response

$\begin{array}{lrrrr}\text { Term } & \text { Coef } & \text { SE Coef } & \text { T } & \text { P } \\ \text { Constant } & 93.8683 & 0.9561 & 98.180 & 0.000 \\ \mathrm{X} 1 & 1.5434 & 0.4622 & 3.339 & 0.002 \\ \mathrm{X} 2 & 3.2736 & 0.4622 & 7.083 & 0.000 \\ \mathrm{X} 3 & 6.2868 & 0.4622 & 13.601 & 0.000 \\ \mathrm{X} 4 & -4.0695 & 0.4622 & -8.804 & 0.000 \\ \mathrm{X} 5 & 0.4583 & 0.4622 & 0.992 & 0.329 \\ \mathrm{X} 1 * \mathrm{X} 1 & -6.3800 & 0.3976 & -16.045 & 0.000 \\ \mathrm{X} 2 * \mathrm{X} 2 & -1.2784 & 0.3976 & -3.215 & 0.003 \\ \mathrm{X} 3 * \mathrm{X} 3 & -2.9065 & 0.3976 & -7.310 & 0.000 \\ \mathrm{X} 4 * \mathrm{X} 4 & -0.4452 & 0.3976 & -1.120 & 0.271 \\ \mathrm{X} 5 * \mathrm{X} 5 & -1.9737 & 0.3976 & -4.964 & 0.000 \\ \mathrm{X} 1 * \mathrm{X} 2 & 0.2524 & 0.5378 & 0.469 & 0.642 \\ \mathrm{X} 1 * \mathrm{X} 3 & -0.5012 & 0.5378 & -0.932 & 0.359 \\ \mathrm{X} 1 * \mathrm{X} 4 & 0.0217 & 0.5378 & 0.040 & 0.968 \\ \mathrm{X} 1 * \mathrm{X} 5 & 0.1760 & 0.5378 & 0.327 & 0.746 \\ \mathrm{X} 2 * \mathrm{X} 3 & -0.0712 & 0.5378 & -0.132 & 0.896 \\ \mathrm{X} 2 * \mathrm{X} 4 & 0.3489 & 0.5378 & 0.649 & 0.521 \\ \mathrm{X} 2 * \mathrm{X} 5 & -0.1558 & 0.5378 & -0.290 & 0.774 \\ \mathrm{X} 3 * \mathrm{X} 4 & 2.0105 & 0.5378 & 3.739 & 0.001 \\ \mathrm{X} 3 * \mathrm{X} 5 & 0.0739 & 0.5378 & 0.137 & 0.892 \\ \mathrm{X} 4 * \mathrm{X} 5 & 0.0644 & 0.5378 & 0.120 & 0.905\end{array}$

$S=3.04198 \quad$ PRESS $=1227.20$

$\mathrm{R}-\mathrm{Sq}=95.428 \quad \mathrm{R}-\mathrm{Sq}($ pred $)=80.41 \% \quad \mathrm{R}-\mathrm{Sq}(\mathrm{adj})=92.46 \%$

Analysis of Variance for Response

$\begin{array}{lrrrrrr}\text { Source } & \text { DF } & \text { Seq SS } & \text { Adj SS } & \text { Adj MS } & \text { F } & \text { P } \\ \text { Regression } & 20 & 5976.07 & 5976.07 & 298.80 & 32.29 & 0.000\end{array}$ $\begin{array}{lrrrrrr}\text { Linear } & 20 & 5976.07 & 5976.07 & 298.80 & 32.29 & 0.000 \\ \text { L } & 5 & 3005.72 & 3005.72 & 601.14 & 64.96 & 0.000\end{array}$

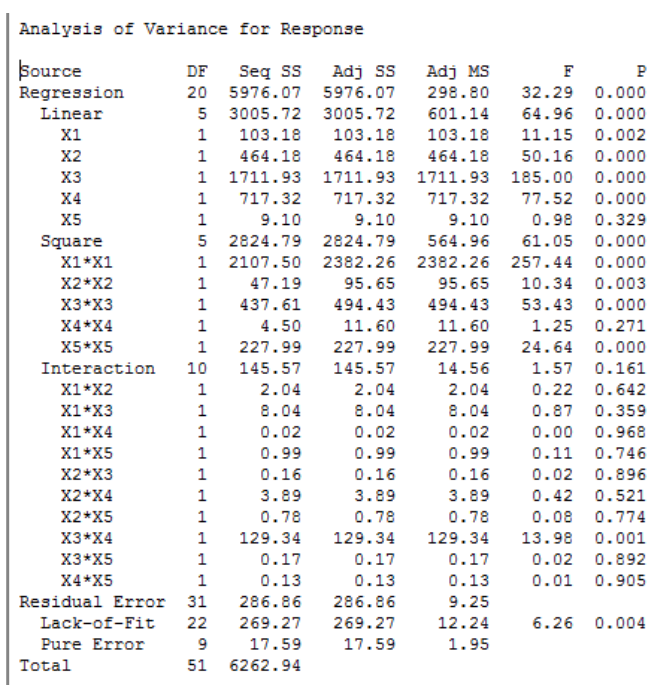

Unusual Observations for Response

$\begin{array}{rrrrrrr}\text { Obs } & \text { Stdorder } & \text { Response } & \text { Fit } & \text { SE Fit } & \text { Residual } & \text { St Resid } \\ 6 & 6 & 81.813 & 86.345 & 2.041 & -4.533 & -2.01 \mathrm{R} \\ 35 & 35 & 84.424 & 78.850 & 2.276 & 5.573 & 2.76 \mathrm{R} \\ 37 & 37 & 58.277 & 62.474 & 2.276 & -4.197 & -2.08 \mathrm{R} \\ 38 & 38 & 99.602 & 92.379 & 2.276 & 7.223 & 3.58 \mathrm{R} \\ 40 & 40 & 85.726 & 81.671 & 2.276 & 4.055 & 2.01 \mathrm{R} \\ 42 & 42 & 88.795 & 83.794 & 2.276 & 5.002 & 2.48 \mathrm{R}\end{array}$

$\mathrm{R}$ denotes an observation with a large standardized residual. 
Estimated Regression Coefficients for response using data in unco

$\begin{array}{lr}\text { Term } & \text { Coef } \\ \text { Constant } & -65.9923 \\ \mathrm{X} 1 & 25.5027 \\ \mathrm{X} 2 & 0.517412 \\ \mathrm{X} 3 & 28.6660 \\ \mathrm{X} 4 & -0.122617 \\ \mathrm{X} 5 & 0.0671769 \\ \mathrm{X} 1 * \mathrm{X} 1 & -1.85272 \\ \mathrm{X} 2 * \mathrm{X} 2 & -0.00833916 \\ \mathrm{X} 3 * \mathrm{X} 3 & -4.18942 \\ \mathrm{X} 4 * \mathrm{X} 4 & -0.00372116 \\ \mathrm{X} 5 * \mathrm{X} 5 & -5.28652 \mathrm{E}-05 \\ \mathrm{X} 1 * \mathrm{X} 2 & 0.000731314 \\ \mathrm{X} 1 * \mathrm{X} 3 & -0.0377430 \\ \mathrm{X} 1 * \mathrm{X} 4 & -0.0106772 \\ \mathrm{X} 1 * \mathrm{X} 5 & -2.24801 \mathrm{E}-05 \\ \mathrm{X} 2 * \mathrm{X} 3 & 0.0538510 \\ \mathrm{X} 2 * \mathrm{X} 4 & 0.00385651 \\ \mathrm{X} 2 * \mathrm{X} 5 & -7.72475 \mathrm{E}-05 \\ \mathrm{X} 3 * \mathrm{X} 4 & 0.0202038 \\ \mathrm{X} 3 * \mathrm{X} 5 & 0.000143868 \\ \mathrm{X} 4 * \mathrm{X} 5 & -8.63207 \mathrm{E}-05\end{array}$

\section{Adsorption of $\mathrm{Pb}^{+2}$ in batch presses}

Response Surface Regression: Response versus X1, X2, X3,

The analysis was done using coded units.

Estimated Regression Coefficients for Response

Term Coef SE Coef T P

Constant $88.8274 \quad 1.3666 \quad 64.997 \quad 0.000$

$\begin{array}{lllll}\mathrm{X} 1 & -1.5632 & 0.6607 & -2.366 & 0.024\end{array}$

$\begin{array}{lrrrr}\mathrm{X} 2 & 1.6083 & 0.6607 & 2.434 & 0.021\end{array}$

$\begin{array}{lllll}\mathrm{X} 3 & 5.7198 & 0.6607 & 8.657 & 0.000\end{array}$

$\begin{array}{lllll}84 & -4.8223 & 0.6607 & -7.299 & 0.000\end{array}$

$\begin{array}{lllll}\mathrm{X} 5 & 0.8272 & 0.6607 & 1.252 & 0.220\end{array}$

$\begin{array}{lllll}\mathrm{X} 1 * \mathrm{X} 1 & -1.7780 & 0.5684 & -3.128 & 0.004\end{array}$

$\begin{array}{lllll}\mathrm{X} 2 * \mathrm{X} 2 & -0.2387 & 0.5684 & -0.420 & 0.677 \\ \mathrm{X} 3 * \mathrm{X} 3 & -3.6843 & 0.5684 & -6.482 & 0.000\end{array}$

$\begin{array}{rrrrr}\mathrm{X} 4 * \mathrm{X} 4 & 0.3612 & 0.5684 & 0.635 & 0.530\end{array}$

$\begin{array}{lllll}\mathrm{X} 5 * \mathrm{X} 5 & -1.2881 & 0.5684 & -2.266 & 0.031\end{array}$

$\begin{array}{lllll}\mathrm{X} 1 * \mathrm{X} 2 & 0.1346 & 0.7687 & 0.175 & 0.862\end{array}$

$\begin{array}{lllll}\mathrm{X} 1 * \mathrm{X} 3 & -0.2699 & 0.7687 & -0.351 & 0.728\end{array}$

$\begin{array}{lllll}\mathrm{X} 1 * \mathrm{X} 4 & 0.4556 & 0.7687 & 0.593 & 0.558\end{array}$

$\begin{array}{lllll}\mathrm{X} 1 * \mathrm{X} 5 & -0.0747 & 0.7687 & -0.097 & 0.923\end{array}$

$\begin{array}{lllll}\mathrm{X} 2 * \mathrm{X} 3 & 0.1878 & 0.7687 & 0.244 & 0.809\end{array}$

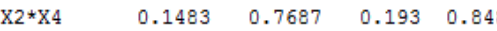

$\begin{array}{lllll}\mathrm{X} 2 \star \mathrm{X} 5 & 0.0539 & 0.7687 & 0.070 & 0.945\end{array}$

$\begin{array}{lllll}\mathrm{X} 3 * \mathrm{X} 4 & 1.5815 & 0.7687 & 2.057 & 0.048\end{array}$

$\begin{array}{lllll}\mathrm{X} 3 * \mathrm{X} 5 & 0.1152 & 0.7687 & 0.150 & 0.882\end{array}$

$\begin{array}{lllll}\mathrm{X} 4 \text { *X5 } & 0.0211 & 0.7687 & 0.027 & 0.978\end{array}$

$S=4.34826 \quad$ PRESS $=2604.31$

R-Sq $=86.56 \%$ R-Sq (pred) $=40.27 \%$ R-Sq (adj) $=77.88$ \%

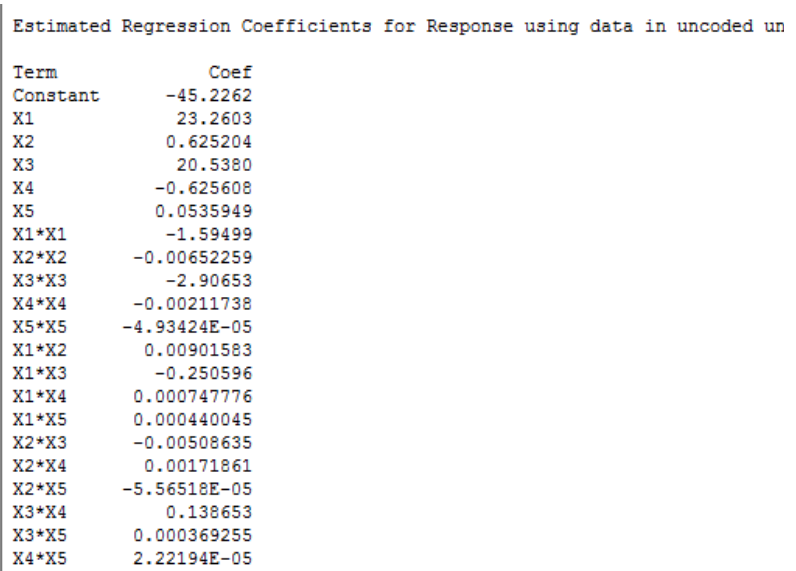

Adsorption of $\mathrm{Zn}^{+2}$ in batch presses

Response Surface Regression: Response versus X1, X2, X3, X4, X The analysis was done using coded units.

Estimated Regression Coefficients for Response

$\begin{array}{lrrrr} & \text { Coef } & \text { SE Coef } & \text { T } & \text { P } \\ \text { Constant } & 73.2958 & 1.8272 & 40.115 & 0.000\end{array}$

$\begin{array}{lllll}\mathrm{X} 1 & 5.4648 & 0.8833 & 6.187 & 0.000\end{array}$

$\begin{array}{lllll}\mathrm{X} 2 & 2.9600 & 0.8833 & 3.351 & 0.002\end{array}$

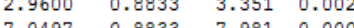

$\begin{array}{lll}7.981 & 0.000\end{array}$

0.0 .0130 .000

$\begin{array}{llll}0.4243 & 0.8833 & 0.480 & 0.634\end{array}$

$\begin{array}{llll}-5.8553 & 0.7599 & -7.705 & 0.000\end{array}$

$\begin{array}{llll}-0.8070 & 0.7599 & -1.062 & 0.296\end{array}$

$\begin{array}{llll}-2.9934 & 0.7599 & -3.939 & 0.000\end{array}$

$\begin{array}{llll}0.6775 & 0.7599 & 0.892 & 0.380\end{array}$

$\begin{array}{llll}-2.3357 & 0.7599 & -3.074 & 0.004\end{array}$

$\begin{array}{llll}0.3611 & 1.0277 & 0.351 & 0.728\end{array}$

$\begin{array}{lll}0.2822 & 1.0277-0.275 & 0.785\end{array}$

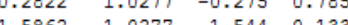

$\begin{array}{llll}1.5062 & 1.0277 & -0.275 & 0.785 \\ -0.536 & 0.133\end{array}$

$\begin{array}{llll}0.3368 & 1.0277 & -0.328 & 0.745\end{array}$

$\begin{array}{llll}0.1665 & 1.0277 & 0.162 & 0.872\end{array}$

$\begin{array}{llll}0.8184 & 1.0277 & 0.796 & 0.432\end{array}$

$\begin{array}{llll}0.0219 & 1.0277 & 0.021 & 0.983\end{array}$

$\begin{array}{llll}-1.1483 & 1.0277 & -1.117 & 0.272\end{array}$

$\begin{array}{llll}0.1949 & 1.0277 & 0.190 & 0.851\end{array}$

$\begin{array}{lllll}\mathrm{X} 4 * \mathrm{X} 5 & 0.2779 & 1.0277 & 0.270 & 0.789\end{array}$

$\begin{array}{lll}S=5.81350 & \text { PRESS }=4679.38 \\ \text { R-Sq }=90.528 & \text { R-Sq (pred) }=57.648 & \text { R-Sq }(\text { adj })=84.40 \%\end{array}$ 


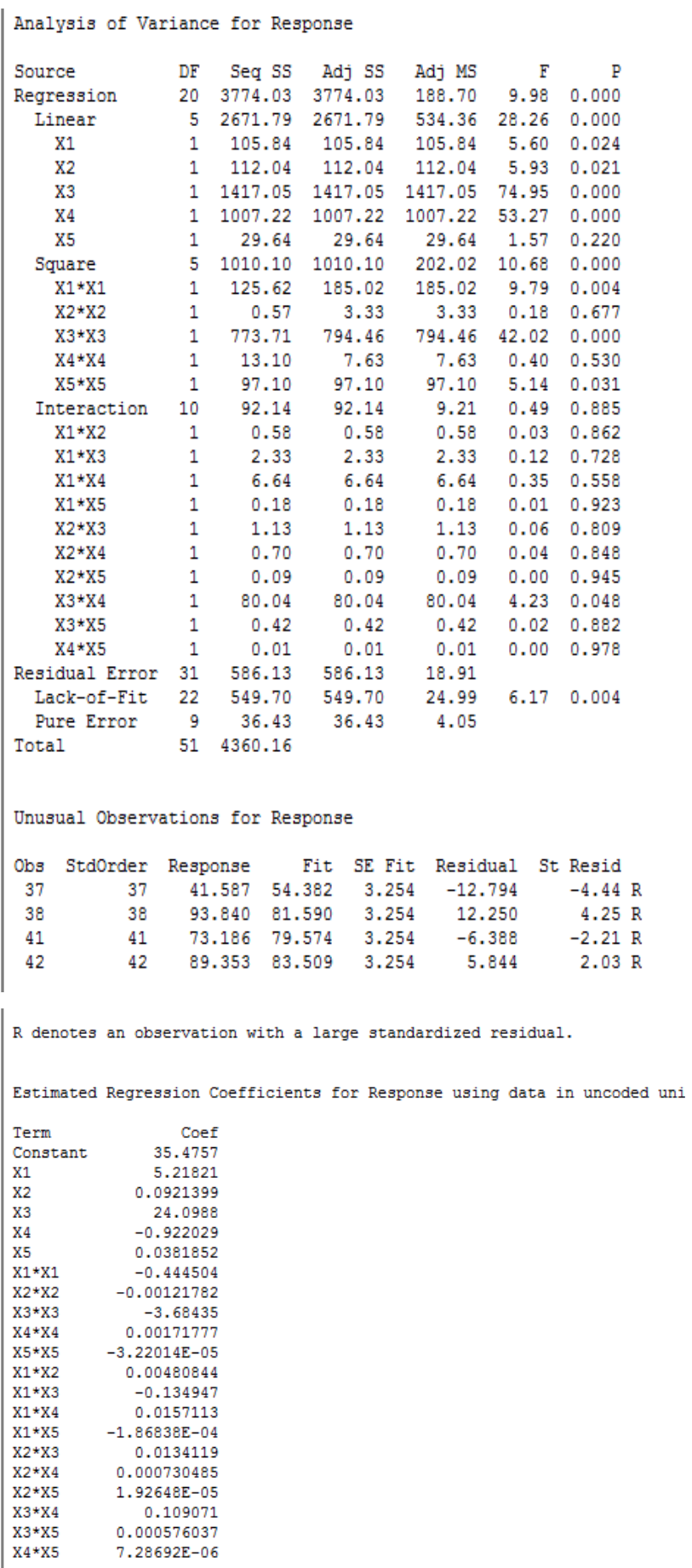

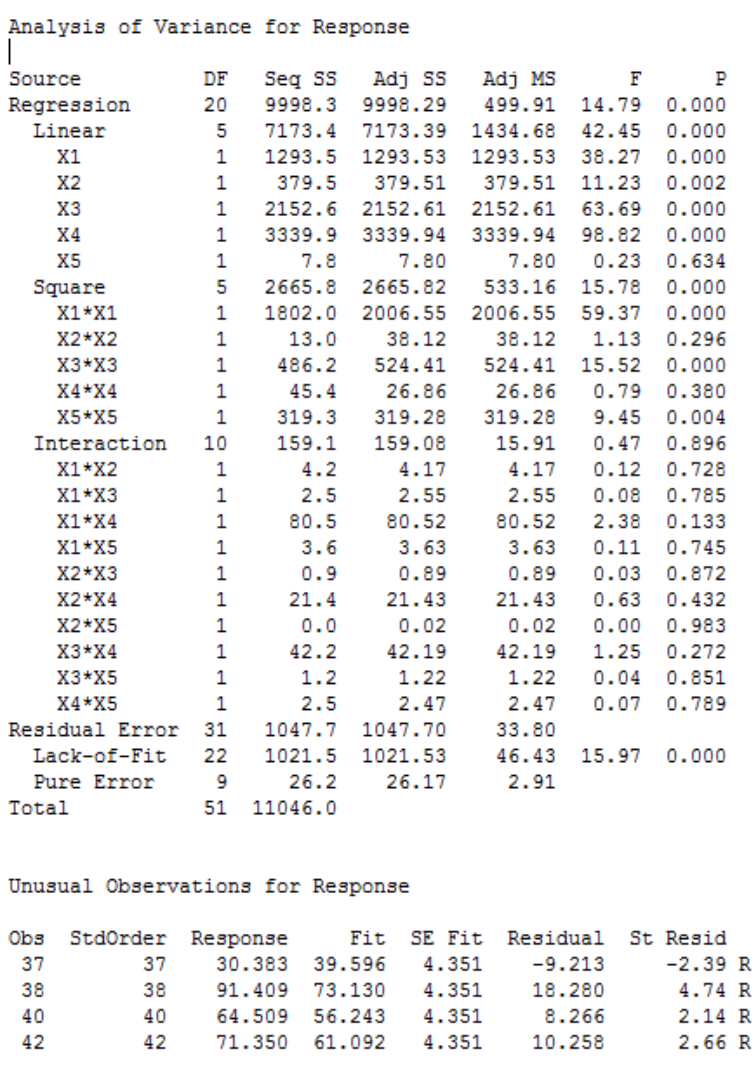

Unusual Observations for Response

$\begin{array}{rrrrrrr}\text { Obs } & \text { Stdorder } & \text { Response } & \text { Fit } & \text { SE Fit } & \text { Residual } & \text { St Resid } \\ 37 & 37 & 30.383 & 39.596 & 4.351 & -9.213 & -2.39 \mathrm{R} \\ 38 & 38 & 91.409 & 73.130 & 4.351 & 18.280 & 4.74 \mathrm{R} \\ 40 & 40 & 64.509 & 56.243 & 4.351 & 8.266 & 2.14 \mathrm{R} \\ 42 & 42 & 71.350 & 61.092 & 4.351 & 10.258 & 2.66 \mathrm{R}\end{array}$

$\mathrm{R}$ denotes an observation with a large standardized residual.

Estimated Regression Coefficients for Response using data in uncoded unit

$\begin{array}{lr}\text { Term } & \text { Coef } \\ \text { Constant } & -59.5905 \\ \mathrm{X} 1 & 21.7061 \\ \mathrm{X} 2 & 0.234516 \\ \mathrm{X} 3 & 27.8446 \\ \mathrm{X} 4 & -1.17755 \\ \mathrm{X} 5 & 0.0656383 \\ \mathrm{X} 1 * \mathrm{X} 1 & -1.46382 \\ \mathrm{X} 2 * \mathrm{X} 2 & -0.00411741 \\ \mathrm{X} 3 * \mathrm{X} 3 & -2.99337 \\ \mathrm{X} 4 * \mathrm{X} 4 & 0.00322231 \\ \mathrm{X} 5 * \mathrm{X} 5 & -5.83916 \mathrm{E}-05 \\ \mathrm{X} 1 * \mathrm{X} 2 & 0.0128980 \\ \mathrm{X} 1 * \mathrm{X} 3 & -0.141076 \\ \mathrm{X} 1 * \mathrm{X} 4 & 0.0546980 \\ \mathrm{X} 1 * \mathrm{X} 5 & -8.41884 \mathrm{E}-04 \\ \mathrm{X} 2 * \mathrm{X} 3 & 0.0118893 \\ \mathrm{X} 2 * \mathrm{X} 4 & 0.00403162 \\ \mathrm{X} 2 * \mathrm{X} 5 & 7.80374 \mathrm{E}-06 \\ \mathrm{X} 3 * \mathrm{X} 4 & -0.0791903 \\ \mathrm{X} 3 * \mathrm{X} 5 & 0.000974679 \\ \mathrm{X} 4 * \mathrm{X} 5 & 9.58348 \mathrm{E}-05\end{array}$

\section{Copyright Disclaimer}

Copyright reserved by the author(s).

This article is an open-access article distributed under the terms and conditions of the Creative Commons Attribution license (http://creativecommons.org/licenses/by/3.0/). 